\title{
Combined use of amentoflavone and ledipasvir could interfere with binding of spike glycoprotein of SARS-CoV-2 to ACE2: the results of molecular docking study
}

\author{
Kateryna V. Miroshnychenko ${ }^{1}$, Anna V. Shestopalova ${ }^{1,2}$ \\ ${ }^{1}$ O.Ya. Usikov Institute for Radiophysics and Electronics of NAS of Ukraine, \\ 12 Ac. Proskura St., Kharkiv, 61085, Ukraine \\ ${ }^{2}$ V.N. Karazin Kharkiv National University, 4 Svobody Sq., Kharkiv, 61022, Ukraine \\ e-mail: kateryna.miroshnychenko@gmail.com \\ Author's ORCID ID \\ K. V. Miroshnychenko ID 0000-0002-2543-6519 \\ A.V. Shestopalova ID 0000-0001-7613-7212
}

\begin{abstract}
The rapid spread of the COVID-19 disease all around the world necessitates the search for fast and effective treatment. The repurposing of the existing drugs seems like the best solution in this situation. In this study the molecular docking method was used to test 248 drugs related to the virus research against spike glycoprotein of SARS-CoV-2, which is responsible for viral entry into the cell. According to the docking results, the best-scored ligand is the natural biflavonoid amentoflavone. Nine of twelve top-ranked ligands are drugs used for hepatitis $\mathrm{C}$ treatment. Among them are ledipasvir, paritaprevir, elbasvir, simeprevir, velpatasvir, glecaprevir and pibrentasvir. The binding sites of the two first-ranked ligands (amentoflavone and ledipasvir) are different. Therefore their combined use may be even more effective in preventing binding of spike glycoprotein to ACE2 than the separate use. Our results suggest that amentoflavone and ledipasvir are promising drug candidates for testing in vitro and in vivo against SARS-CoV-2.
\end{abstract}

Keywords: SARS-CoV-2; COVID-19; spike glycoprotein; molecular docking; amentoflavone; ledipasvir; hepatitis $\mathrm{C}$ drugs

\section{Introduction}

Starting from December 2019 the COVID-19 disease caused by severe acute respiratory syndrome coronavirus 2 (SARS-CoV-2) is rapidly spreading around the world. On the 27th of May there were more than 5,609,000 cases and 350,800 deaths of COVID-19 [1]. Currently there are no vaccines nor specific antiviral treatments for COVID-19 [2]. The phylogenetic analysis of SARS-CoV-2 revealed that it belongs to the betacoronavirus genus and is closely related to the bat coronaviruses and human SARS-CoV [3, 4]. To enter the host cell SARS-CoV-2 uses its spike glycoprotein to bind to the angiotensin converting enzyme 2 (ACE2) receptor [5]. This makes SARS-CoV-2 spike glycoprotein an attractive target for drug development: ligands that bind to the spike glycoprotein-ACE2 contact surface could possibly interfere with the ACE2-spike interaction and prevent the penetration of the virus into the cell. Considering that the development of new drugs to COVID-19 could take years the repurposing of the already existing drugs looks like a good alternative.

In this study we used molecular docking method to test the list of 248 BLDPharm drugs which are related to the virus research against spike glycoprotein of SARS-CoV-2. For ten top-ranked drugs the binding sites and interactions with spike glycoprotein were analyzed in detail.

\section{Computational Methods}

The receptor binding domain (RBD) of spike glycoprotein of SARS-CoV-2 was chosen as a docking target. Its structure was taken from the RBD/ACE2-B0AT1 complex deposited in PDB [6] (PDB ID $6 \mathrm{M} 17$, chain $\mathrm{E}$ ). The protonation state of residues at $\mathrm{pH}=7$ was checked using propka3.1 $[7,8]$ and the 
missing hydrogens were added using LEaP from AmberTools19 [9]. Then the pdbqt-file of proteintarget was prepared in AutoDockTools 1.5.6 [10].

The 248 BLDPharm drugs related to the virus research (Supplementary material, Table S1) were searched by their BD codes or CAS numbers in the ZINC15 database [11] or PubChem [12]. Files in mol2 or sdf format were downloaded. For some drugs there were several stereoisomers in ZINC15 so the total number of ligand files raised up to 327 . The files of sdf format were converted to the mol2 format using Open Babel 2.4.1 [13]. And finally, the pdbqt ligand files were prepared from mol2 files using prepare_ligand4.py script of AutoDockTools 1.5.6 [10].

The molecular docking was carried out in AutoDock Vina 1.1.2 [14]. The search space was a rectangular box $40 \AA \times 40 \AA \times 28 \AA$ that was centered at $(170,117,240)$. In PDB ID 6 M17 complex, this box covers all ACE2 heavy atoms that are within $6 \AA$ of RBD (Fig. 1). Due to the large size of the search space, the exhaustiveness parameter of AutoDock Vina was raised to 50.

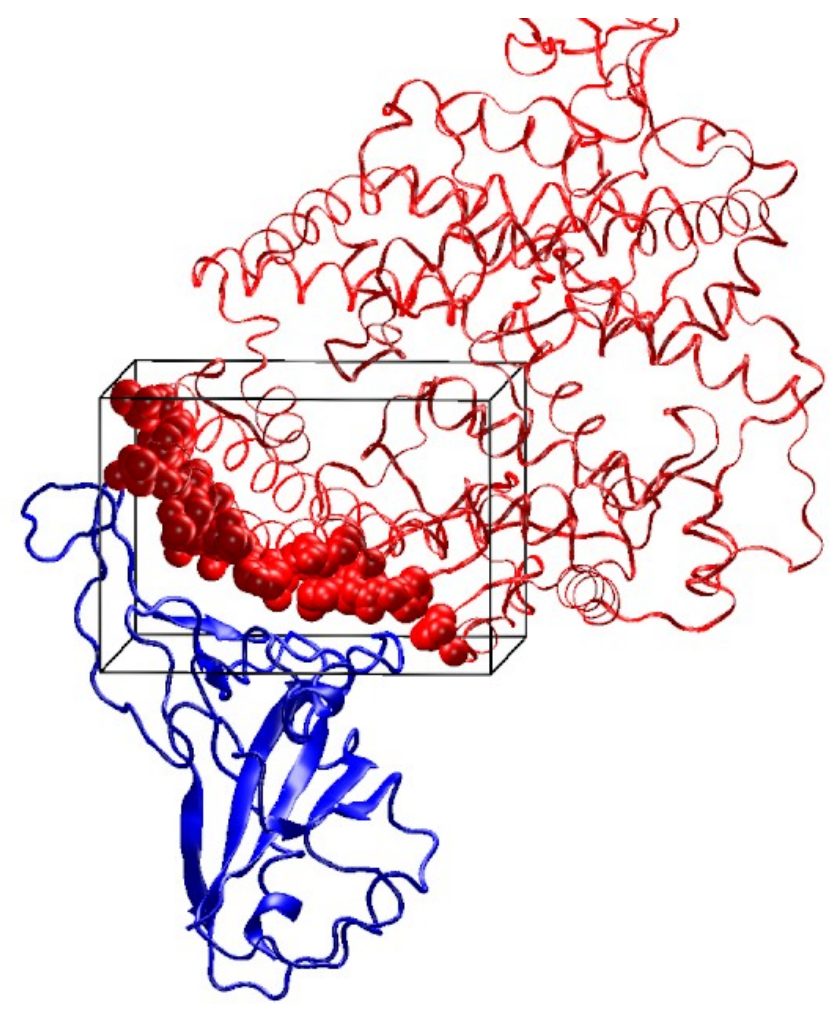

Fig. 1 The location of the AutoDock Vina search box. RBD is shown with blue color. ACE2 is red. Atoms of ACE2 that are within $6 \AA$ of RBD are shown with red van der Waals spheres. The search space is black rectangular box.

The visualization of docking results was done in VMD-1.9.3 [15]. The 3D and 2D contact maps between ligands and binding site residues of RBD were built in PLIP tool [16] and ligplot+ [17], respectively. Maximum acceptable distance between hydrogen bond acceptor and donor was set to $3.35 \AA$. The minimum angle between hydrogen bond donor, hydrogen and acceptor was equal to $90^{\circ}$. The hydrophobic interactions were defined for hydrophobic atoms (C or S) that were within $3.9 \AA$ of each other.

\section{Results and discussion}

The twelve top-ranked ligands are listed in Table 1. Free energies of binding predicted for them by AutoDock Vina are in range $-8.5--7.7 \mathrm{kcal} / \mathrm{mol}$. Most top-ranked ligands are large hydrophobic 
molecules. Molecular weight is greater than $700 \mathrm{~g} / \mathrm{mol}$ for 10 of 12 top-ranked ligands with the exception of amentoflavone $(\mathrm{M}=538 \mathrm{~g} / \mathrm{mol})$ and sotrastaurin $(\mathrm{M}=438 \mathrm{~g} / \mathrm{mol})$.

Table 1. Top-ranked ligands

\begin{tabular}{|l|l|l|l|l|l|l|}
\hline Rank & ZINC_ID & $\begin{array}{l}\text { Vina } \\
\text { energy, } \\
\text { kcal/mol }\end{array}$ & Name & BD index & M, g/mol & $\log$ \\
\hline 1 & ZINC000003984030 & -8.5 & Amentoflavone & BD113814 & 538.464 & 5.134 \\
\hline 2 & ZINC000150338819 & -8.4 & Ledipasvir & BD290997 & 889.017 & 8.607 \\
\hline 3 & ZINC000197964623 & -8.3 & Paritaprevir & BD304034 & 765.893 & 3.637 \\
\hline 4 & ZINC000150588351 & -8.2 & Elbasvir & BD630189 & 882.035 & 8.116 \\
\hline 5 & ZINC000085540268 & -7.9 & Simeprevir & BD306053 & 749.956 & 6.094 \\
\hline 6 & ZINC000253632968 & -7.9 & Simeprevir stereoisomer 1 & BD306053 & 749.956 & 5.254 \\
\hline 7 & ZINC000203686879 & -7.9 & Velpatasvir & BD629142 & 883.019 & 7.732 \\
\hline 8 & ZINC000150656835 & -7.9 & Simeprevir stereoisomer 2 & BD306053 & 749.956 & 5.254 \\
\hline 9 & ZINC000003973984 & -7.9 & $\begin{array}{l}\text { Sotrastaurin } \\
\text { 3-(1H-Indol-3-yl)-4-(2-(4- } \\
\text { methylpiperazin-1-yl)quinazolin-4- } \\
\text { yl)-1H-pyrrole-2,5-dione }\end{array}$ & BD559085 & 438.491 & 2.43 \\
\hline 10 & ZINC000936069565 & -7.8 & Glecaprevir & BD768426 & 838.878 & 3.857 \\
\hline 11 & ZINC000254124762 & -7.7 & Alpha-glycyrrhizin & BD56100 & 822.942 & 2.246 \\
\hline 12 & NO ZINC ID & -7.7 & Pibrentasvir & BD766533 & 1113.2 & 7.4 \\
\hline
\end{tabular}

$\log \mathrm{P}-$ is the logarithm of octanol-water partition coefficient. It is a measure of hydrophobicity of a compound.

First in ranking is amentoflavone, biflavonoid, a natural product found in Ginkgo biloba and Hypericum perforatum [18]. It is known to be an inhibitor of CYP3A4, CYP2C9 and human cathepsin $\mathrm{B}[19,20]$. There is no information in literature regarding the use of amentoflavone against COVID-19 so far.

Nine of the twelve top-ranked ligands are drugs for hepatitis $\mathrm{C}$ virus (HCV) treatment. Ledipasvir (rank 2), elbasvir (rank 4), velpatasvir (rank 7) and pibrentasvir (rank 12) are inhibitors of NS5A protein of HCV [21]. Paritaprevir (rank 3), simeprevir (rank 5, rank 6 (stereoisomer 1), rank 8 (stereoisomer 2)) and glecaprevir (rank 10) inhibit HCV protease complex comprised of non-structural protein 3 and 4A (NS3/NS4A) [22]. Glecaprevir and pibrentasvir are used in combination (brand names Mavyret and Maviret) to treat HCV [23]. For all of these drugs the successful docking results against one or several SARS-CoV-2 proteins are reported in literature [24-29]. There is also one clinical study in which HCV drug danoprevir was used in combination with ritonavir for treatment of small group of COVID-19 patients (11 people) and all patients recovered [30]. These results are encouraging for the use of HCV drugs in COVID-19 treatment.

Sotrastaurin, ranked ninth, is a potent pan-protein kinase $\mathrm{C}$ inhibitor [31]. It is used in cancer therapy [32] and as an immunosuppressant upon transplantation [33], which calls into question the possibility of its use as an antiviral drug.

Alpha-glycyrrhizin (rank 11) is a derivative of glycyrrhizic acid, which is found in licorice roots and has a variety of biological and pharmacological activities [34]. It is used as a food sweetener and as a traditional medicine in the treatment of liver diseases [35]. Diammonium glycyrrhizinate is one of drugs that are currently undergoing clinical trials against COVID-19 [36]. 
To analyze what forces contribute to ligand-receptor affinity, the 3D and 2D contact maps were built in plip and ligplot+ for top-ranked ligands (Fig. 2 - Fig. 32). The interactions are summarized in Table 2. Since plip and ligplot + determine hydrogen bonds and hydrophobic interactions differently, two sets of values are given. One can see that ligand-RBD complexes are stabilized due to hydrophobic interactions, hydrogen bonds and $\pi$-stacking. TYR489 or TYR505 residues of RBD are involved usually in $\pi$-stacking interactions with ligand. For most ligands, the number of hydrophobic contacts is greater or comparable to the number of hydrogen bonds. Only for alpha-glycyrchizine and glycaprevir, hydrogen bonds make the main contribution to the stabilization of complex with RBD.

The number of RBD residues that interacted with ligand varied from 5 to 9 for top-ranked ligands according to ligplot+ data (Table 2). To compare the binding sites of ligands with the ACE2 binding site on RBD (Fig. 33), we built the 2D contact map of RBD and ACE2 using coordinates of PDBID $6 \mathrm{~m} 17$ [6] (Fig. 34). One can see that ACE2 interacts with seven residues of RBD via two hydrogen bonds (with RBD residues GLN493 and GLY502) and hydrophobic contacts (with RBD residues GLY476, PHE486, TYR489, GLN498, TYR505). None of the top-ranked ligands can overlap all the residues of the ACE2 binding site on RBD. The best result has Simeprevir: it covers three of seven residues of the ACE2 binding site (Table 2). Most top-ranked ligands occupy two residues of RBD which ACE2 binds to. It is interesting that the two first-ranked ligands (amentoflavone and ledipasvir) have different binding sites on RBD (Table 2, Fig. 35), which makes possible their combined use. Considering that amentoflavone is a CYP3A4 and CYP2C9 inhibitor, its use in combination with ledipasvir could possibly increase the plasma concentration of latter. But before clinical trials, the combination of amentoflavone and ledipasvir should be carefully tested first in vitro, then in cell cultures, and finally in vivo.

Table 2. The contacts between ligands and RBD in the docking complexes

\begin{tabular}{|l|l|l|l|l|l|l|}
\hline Rank & Ligand & $\begin{array}{l}\mathrm{N}_{\mathrm{HB}} \\
\mathrm{plip/} \\
\text { ligplot+ }\end{array}$ & $\begin{array}{l}\mathrm{N}_{\text {T-stacking }} \\
\mathrm{N}_{\text {m-cation }} \\
\text { plip }\end{array}$ & $\begin{array}{l}\mathrm{N}_{\text {hydrophobic }} \\
\text { plip/ } \\
\text { ligplot+ }\end{array}$ & $\begin{array}{l}\mathrm{N}_{\text {binding site res }} \\
\text { plip/ } \\
\text { ligplot+ }\end{array}$ & $\begin{array}{l}\text { RBD binding site residues plip/ligplot+ } \\
\text { bold }+ \text { common binding site residues for plip and } \\
\text { ligplot+ } \\
\text { red color }- \text { RBD residues that have contact with } \\
\text { ACE2 }\end{array}$ \\
\hline 1 & Amentoflavone & $4 / 4$ & $2 / 0$ & $2 / 22$ & $6 / 6$ & $\begin{array}{l}\text { TYR453, TYR495, GLY496, GLN498, ASN501, } \\
\text { TYR505/ } \\
\text { ARG403, TYR453, TYR495, GLN498, ASN501, } \\
\text { TYR505 }\end{array}$ \\
\hline 2 & Ledipasvir & $2 / 0$ & $2 / 0$ & $4 / 30$ & $4 / 6$ & $\begin{array}{l}\text { PHE456, TYR489, PHE490, GLN493/ } \\
\text { LEU452, PHE456, ASN487, TYR489, PHE490, } \\
\text { GLN493 }\end{array}$ \\
\hline 3 & Paritaprevir & $5 / 4$ & $0 / 0$ & $5 / 19$ & $8 / 8$ & $\begin{array}{l}\text { ARG403, TYR453, LEU455, GLN493, SER494, } \\
\text { TYR495, PHE497, TYR505/ } \\
\text { ARG403, TYR453, LEU455, GLN493, SER494, } \\
\text { TYR495, PHE497, TYR505 }\end{array}$ \\
\hline 4 & Elbasvir & $2 / 1$ & $2 / 0$ & $11 / 29$ & $7 / 6$ & $\begin{array}{l}\text { PHE456, TYR473, ALA475, TYR489, PHE490, } \\
\text { GLN493, SER494/ } \\
\text { PHE456, TYR473, ALA475, TYR489, PHE490, } \\
\text { SER494 }\end{array}$ \\
\hline
\end{tabular}




\begin{tabular}{|c|c|c|c|c|c|c|}
\hline 5 & Simeprevir & $6 / 5$ & $1 / 0$ & $7 / 22$ & 7/8 & $\begin{array}{l}\text { ARG403, LYS417, TYR453, GLN493, TYR495, } \\
\text { THR500, TYR505/ } \\
\text { ARG403, LYS417, TYR453, GLN493, TYR495, } \\
\text { ASN 501, GLY502, TYR505 }\end{array}$ \\
\hline 7 & Velpatasvir & $3 / 3$ & $0 / 1$ & $5 / 21$ & $6 / 8$ & $\begin{array}{l}\text { ARG403, LYS417, TYR421, TYR495, PHE497, } \\
\text { TYR505/ } \\
\text { ARG403, LYS417, TYR421, LEU455, TYR495, } \\
\text { PHE497, ASN501, TYR505 }\end{array}$ \\
\hline 9 & Sotrastaurin & $0 / 0$ & $2 / 0$ & $2 / 25$ & $3 / 5$ & $\begin{array}{l}\text { PHE497, ASN501, TYR505/ } \\
\text { ARG403, PHE497, GLN498, ASN501, TYR505 }\end{array}$ \\
\hline 10 & Glecaprevir & $5 / 4$ & $0 / 0$ & $1 / 4$ & $5 / 5$ & $\begin{array}{l}\text { ARG403, TYR449, TYR453, TYR495, GLN498/ } \\
\text { ARG403, TYR449, TYR453, GLY496, GLN498 }\end{array}$ \\
\hline 11 & $\begin{array}{l}\text { Alpha- } \\
\text { glycyrrhizin }\end{array}$ & $9 / 6$ & $0 / 0$ & $2 / 9$ & $9 / 6$ & $\begin{array}{l}\text { ARG403, GLN409, LYS417, TYR449, LEU455, } \\
\text { GLN493, SER494, GLY496, GLN498/ } \\
\text { ARG403, LYS417, LEU455, SER494, GLY496, } \\
\text { GLN498 }\end{array}$ \\
\hline 12 & Pibrentasvir & $3 / 1$ & $1 / 0$ & $5 / 22$ & 7/9 & $\begin{array}{l}\text { ARG403, LYS417, TYR453, LEU455, PHE456, } \\
\text { TYR489, GLN493/ } \\
\text { ARG403, LYS417, TYR453, LEU455, PHE456, } \\
\text { GLU484, TYR489, GLN493, TYR495 }\end{array}$ \\
\hline
\end{tabular}

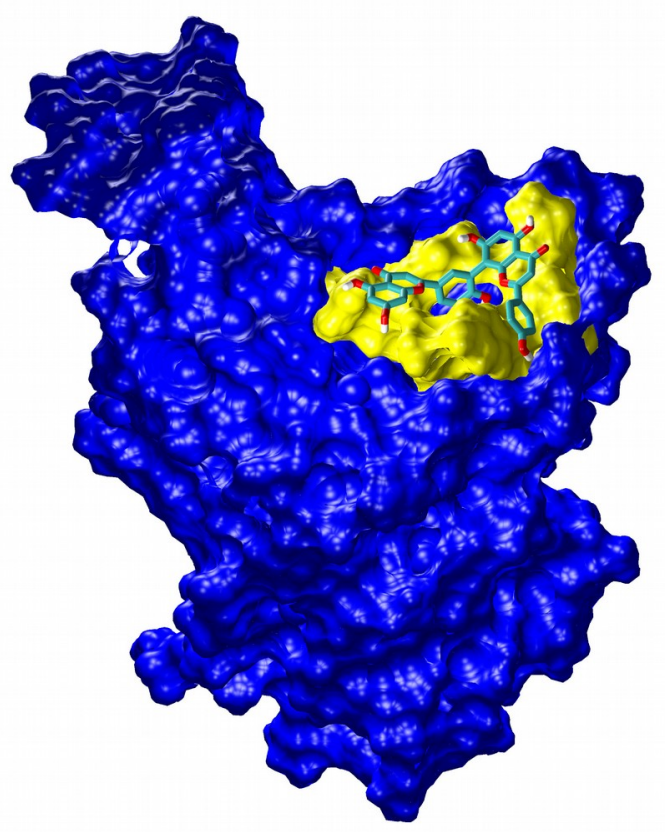

Fig. 2 The location of the amentoflavone binding site in RBD. Residues of RBD interacting with amentoflavone are highlighted in yellow. 


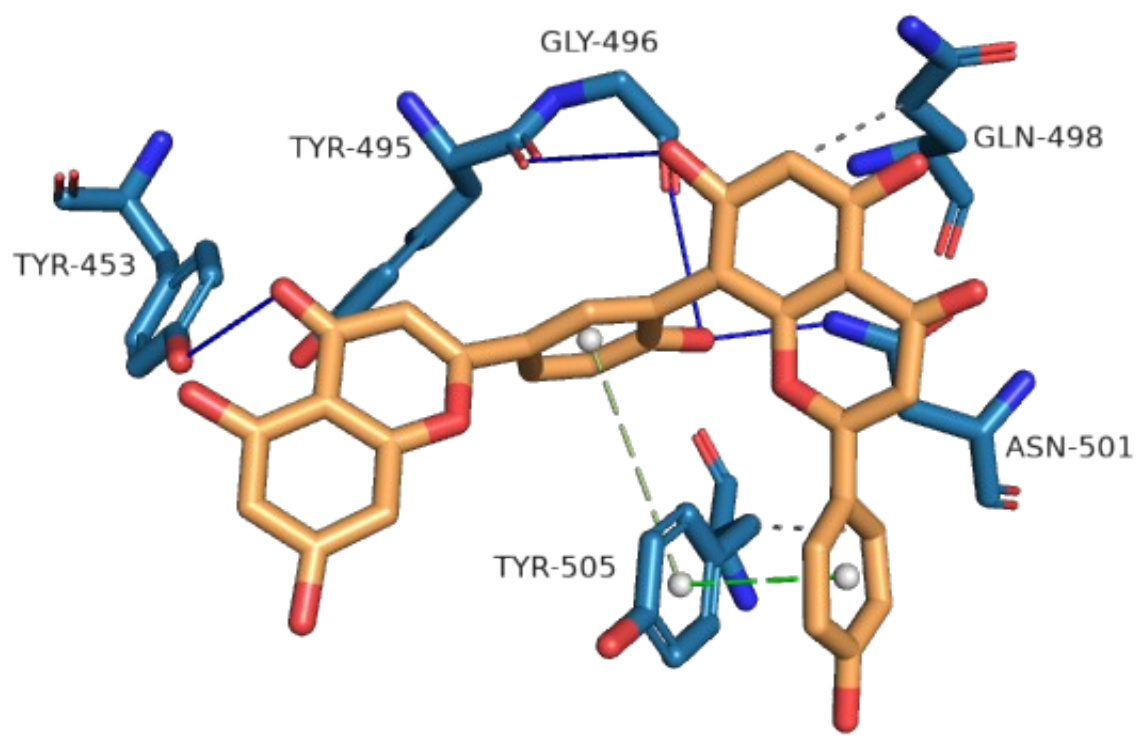

Fig. 3 The 3D contact map of amentoflavone and RBD built in PLIP tool. Hydrogen bonds are shown as blue solid lines, $\pi$-stacking - as green dashed lines, hydrophobic interactions - as gray dotted lines.

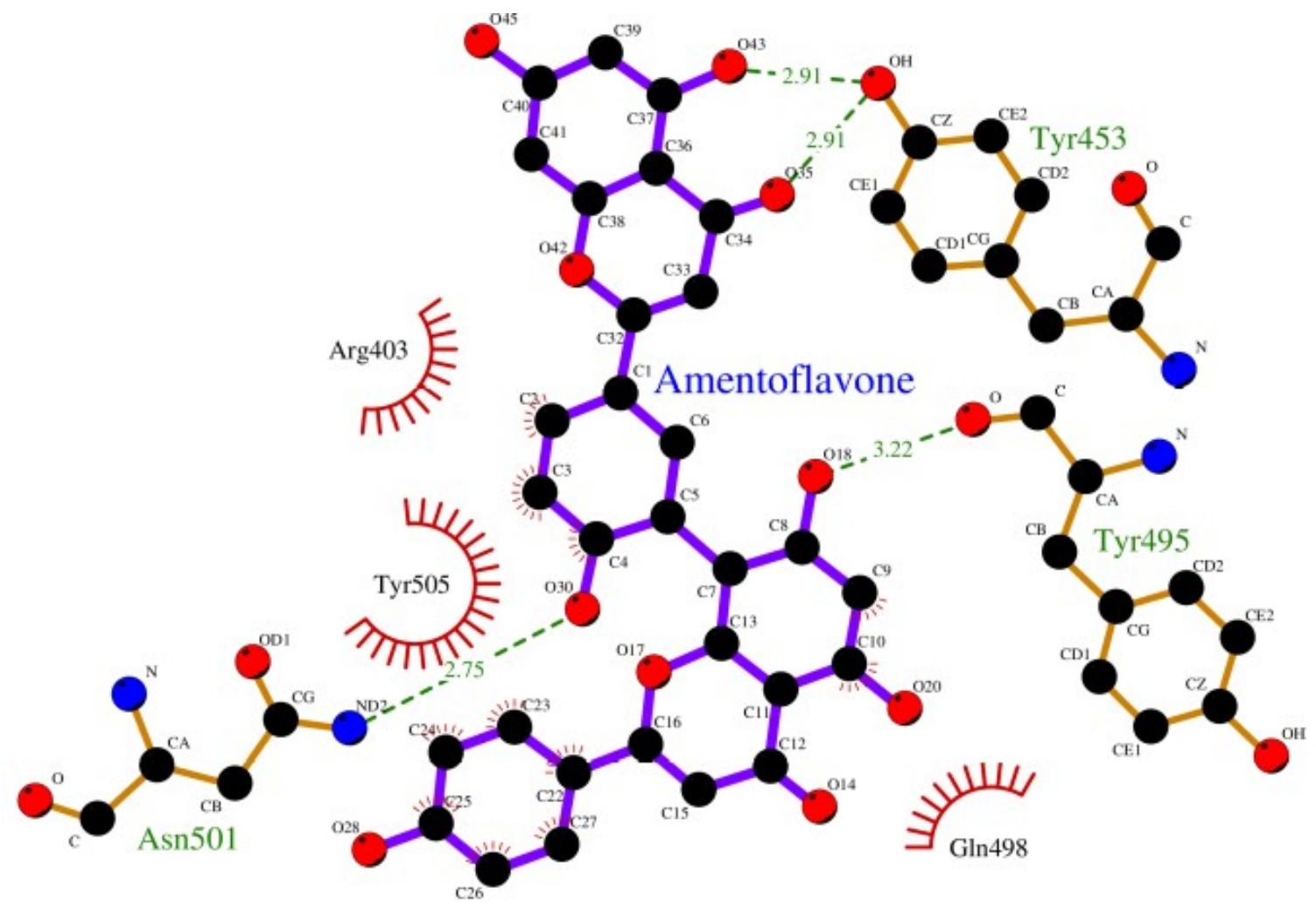

Fig. 4 The 2D contact map of amentoflavone and RBD built in ligplot + . Hydrogen bonds are shown as green dashed lines. Ligand atoms that participate in hydrophobic interactions are surrounded by small red "hedgehogs" (each such atom can form several hydrophobic contacts with different protein atoms). Protein residues involved only in hydrophobic interactions with the ligand (not forming hydrogen bonds with it) are shown as large red "hedgehogs". 


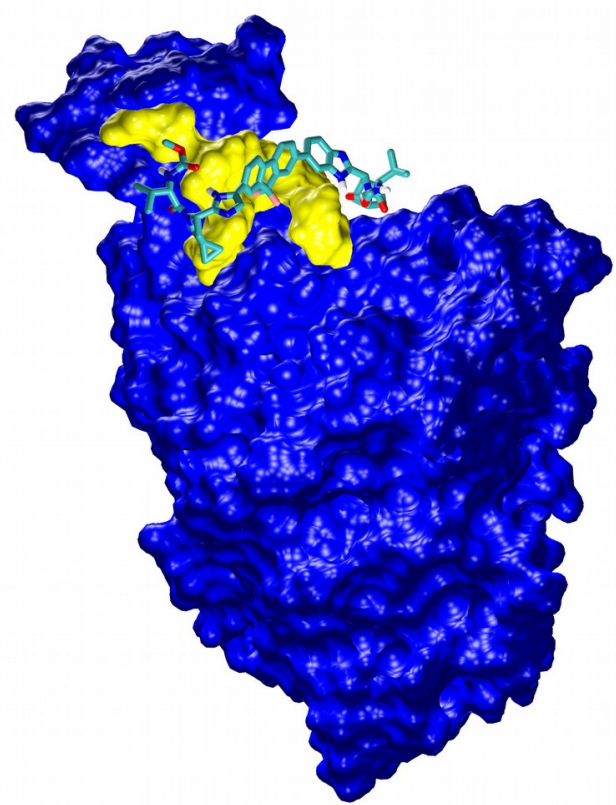

Fig. 5 The location of the ledipasvir binding site in RBD.

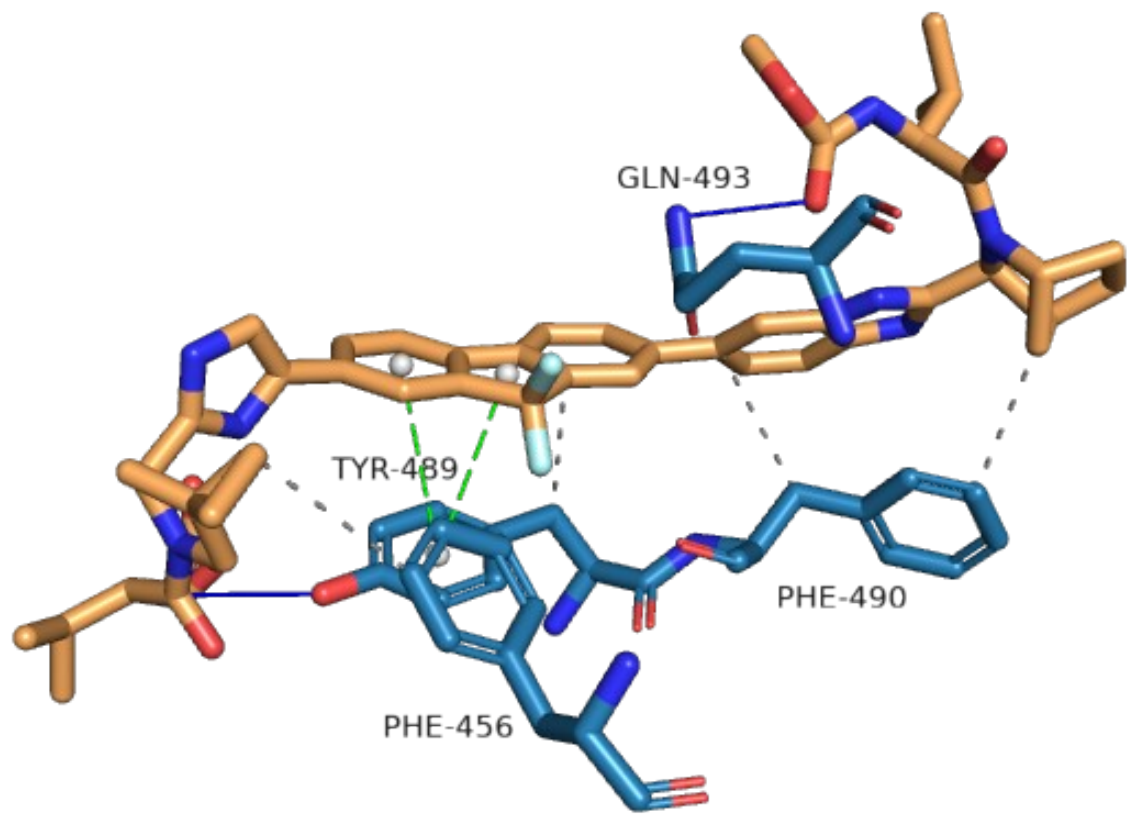

Fig. 6 The 3D contact map of ledipasvir and RBD built in PLIP tool. 


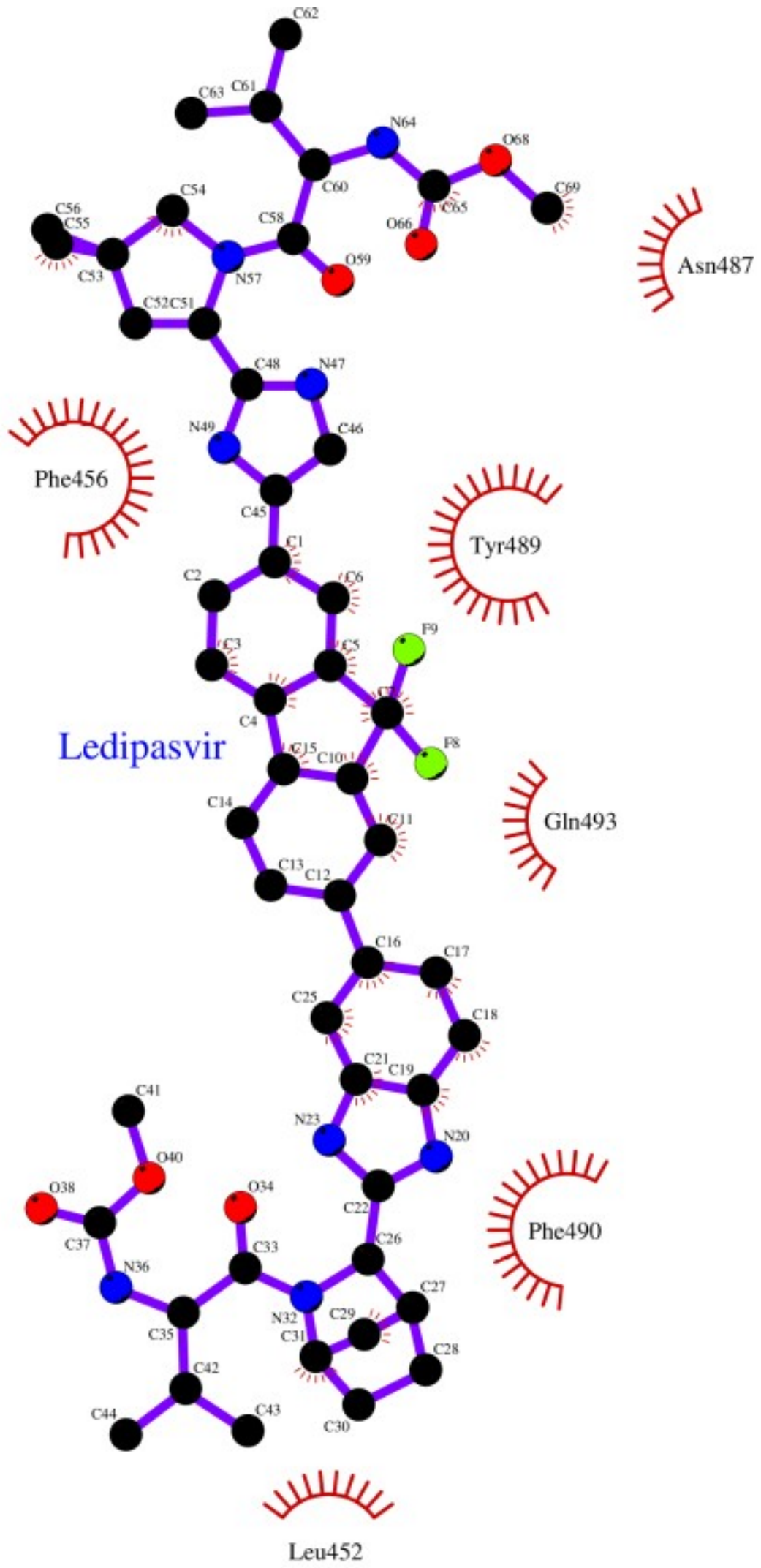

Fig. 7 The 2D contact map of ledipasvir and RBD built in ligplot + . 


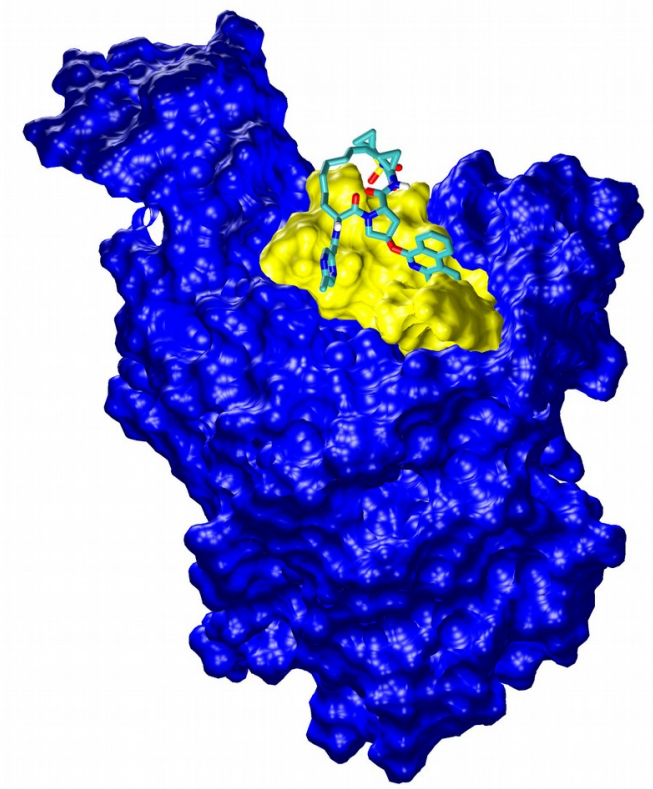

Fig. 8 The location of the paritaprevir binding site in RBD.

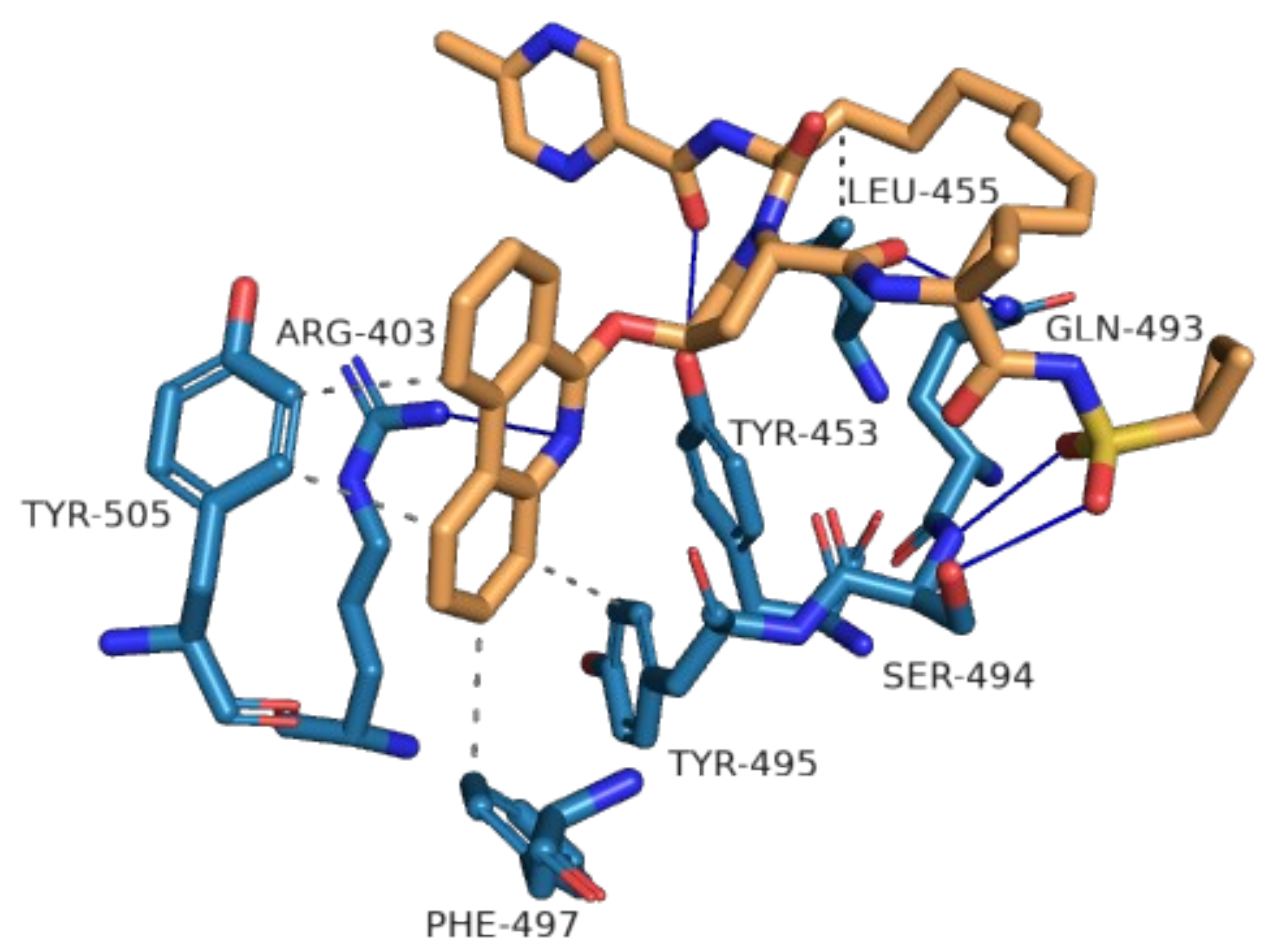

Fig. 9 The 3D contact map of paritaprevir and RBD built in PLIP tool. 


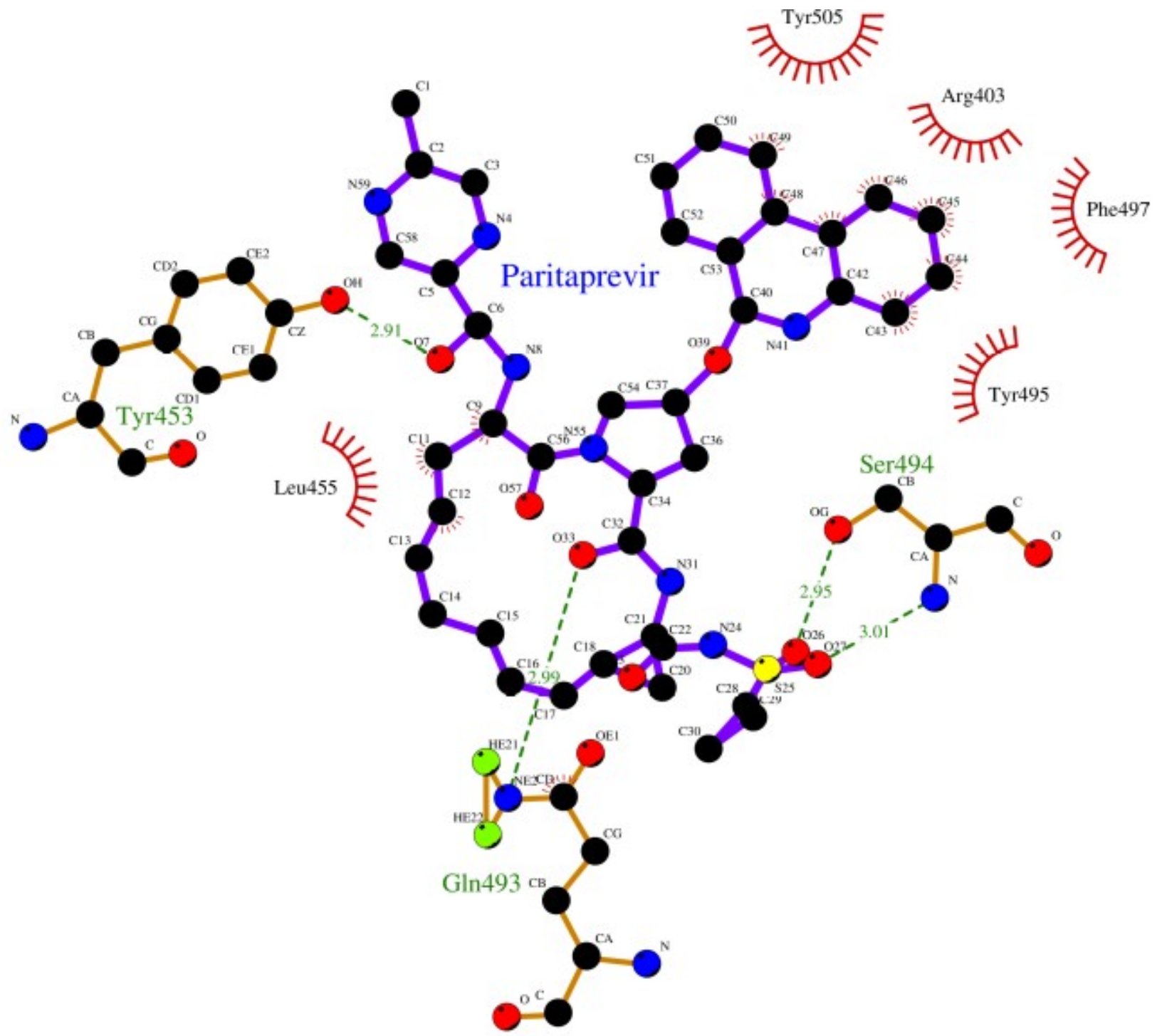

Fig. 10 The 2D contact map of paritaprevir and RBD built in ligplot+. 


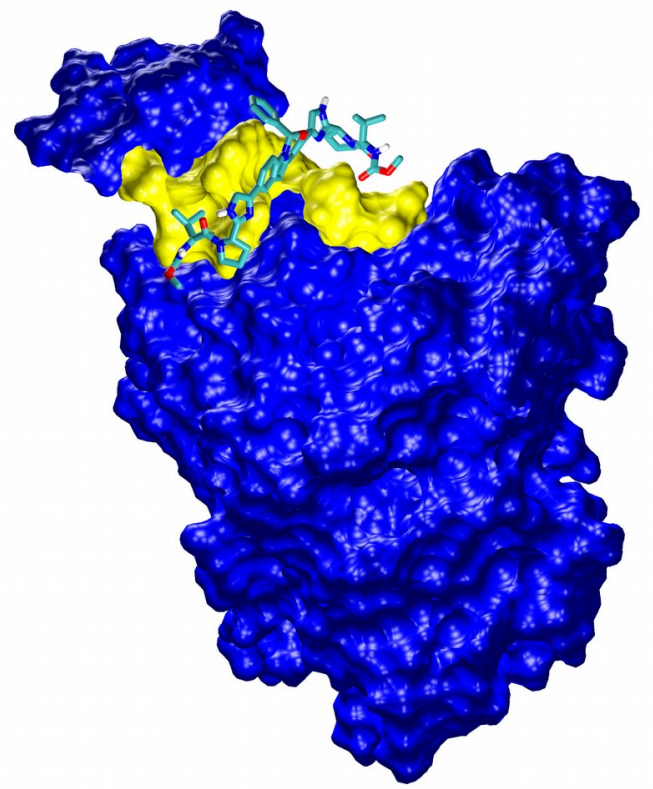

Fig. 11 The location of the elbasvir binding site in RBD.

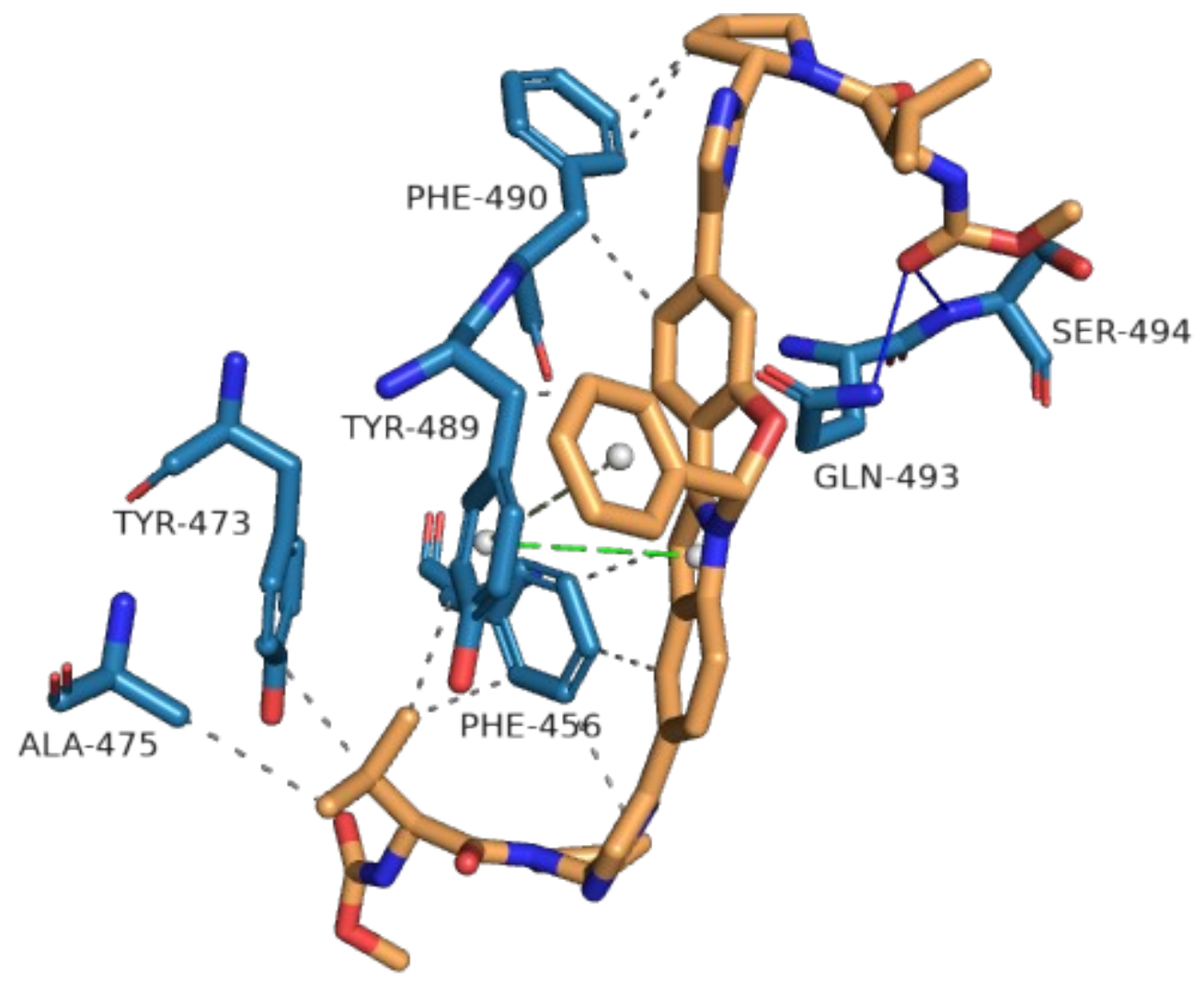

Fig. 12 The 3D contact map of elbasvir and RBD built in PLIP tool. 


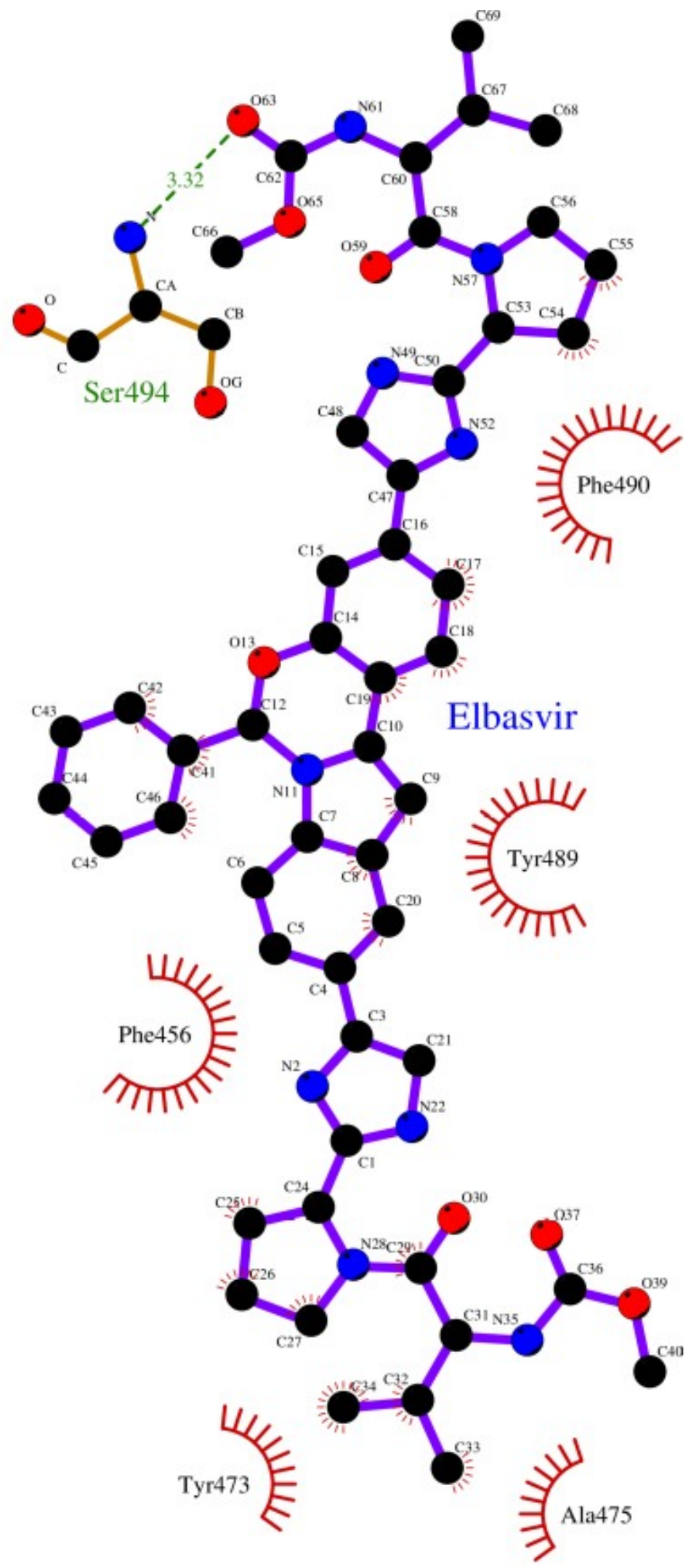

Fig. 13 The 2D contact map of elbasvir and RBD built in ligplot+. 


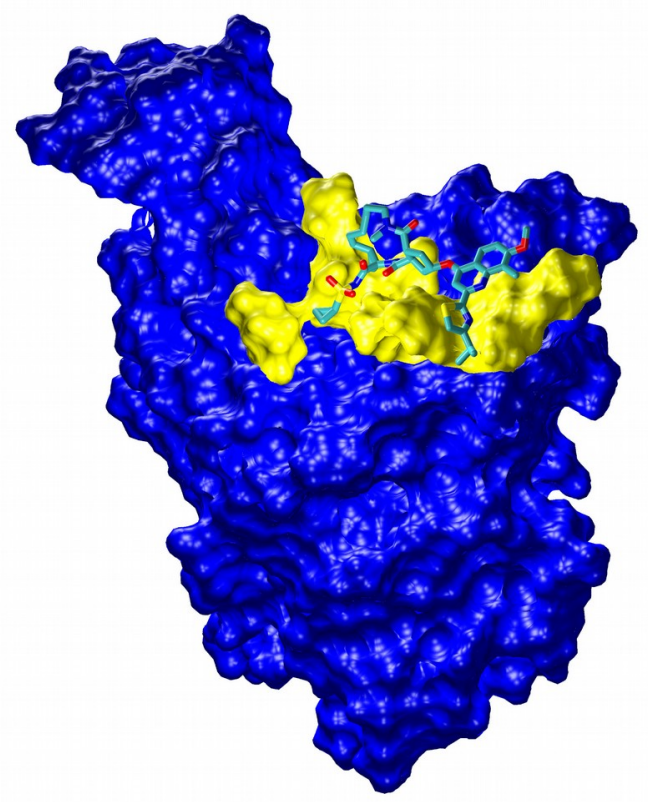

Fig. 14 The location of the simeprevir (ZINC000085540268, rank 5) binding site in RBD.

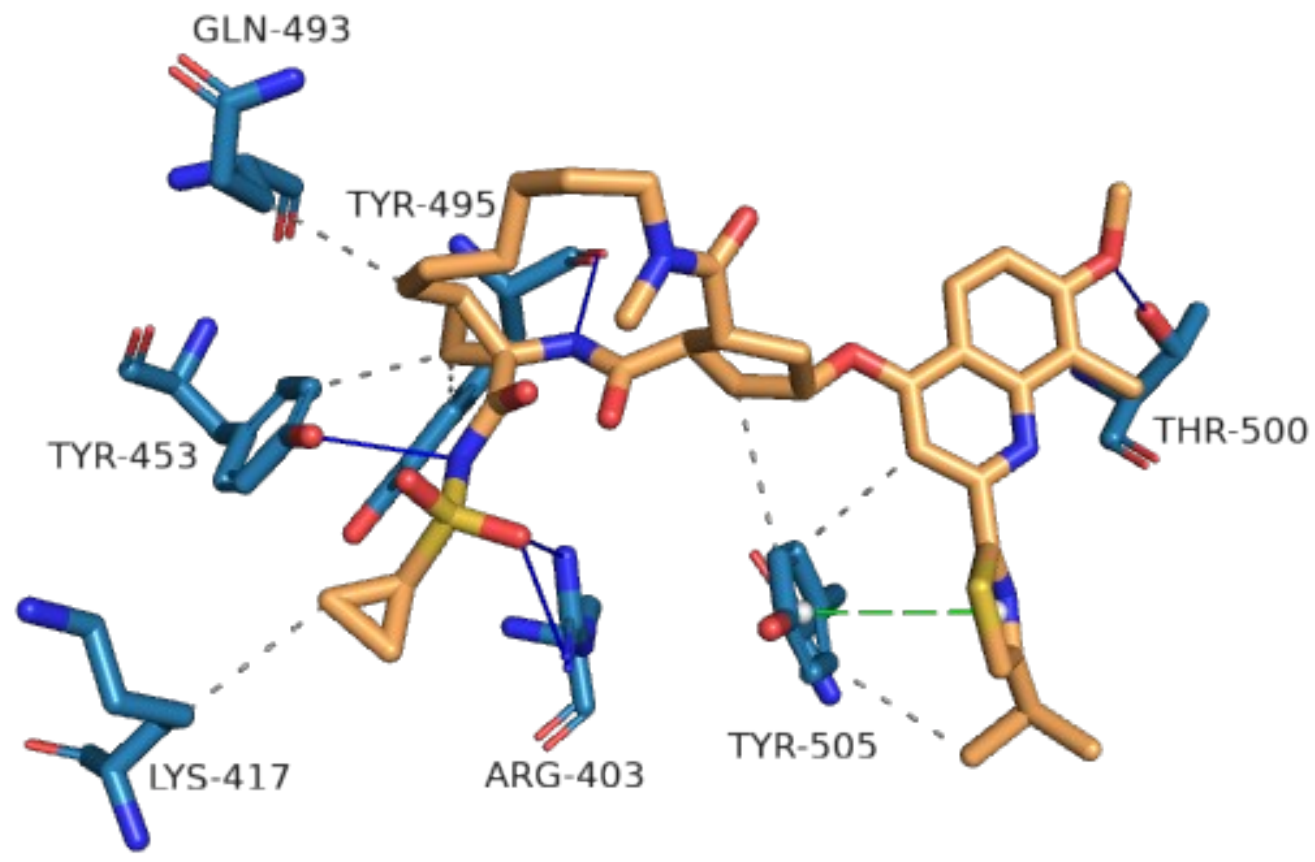

Fig. 15 The 3D contact map of simeprevir (ZINC000085540268) and RBD built in PLIP tool. 


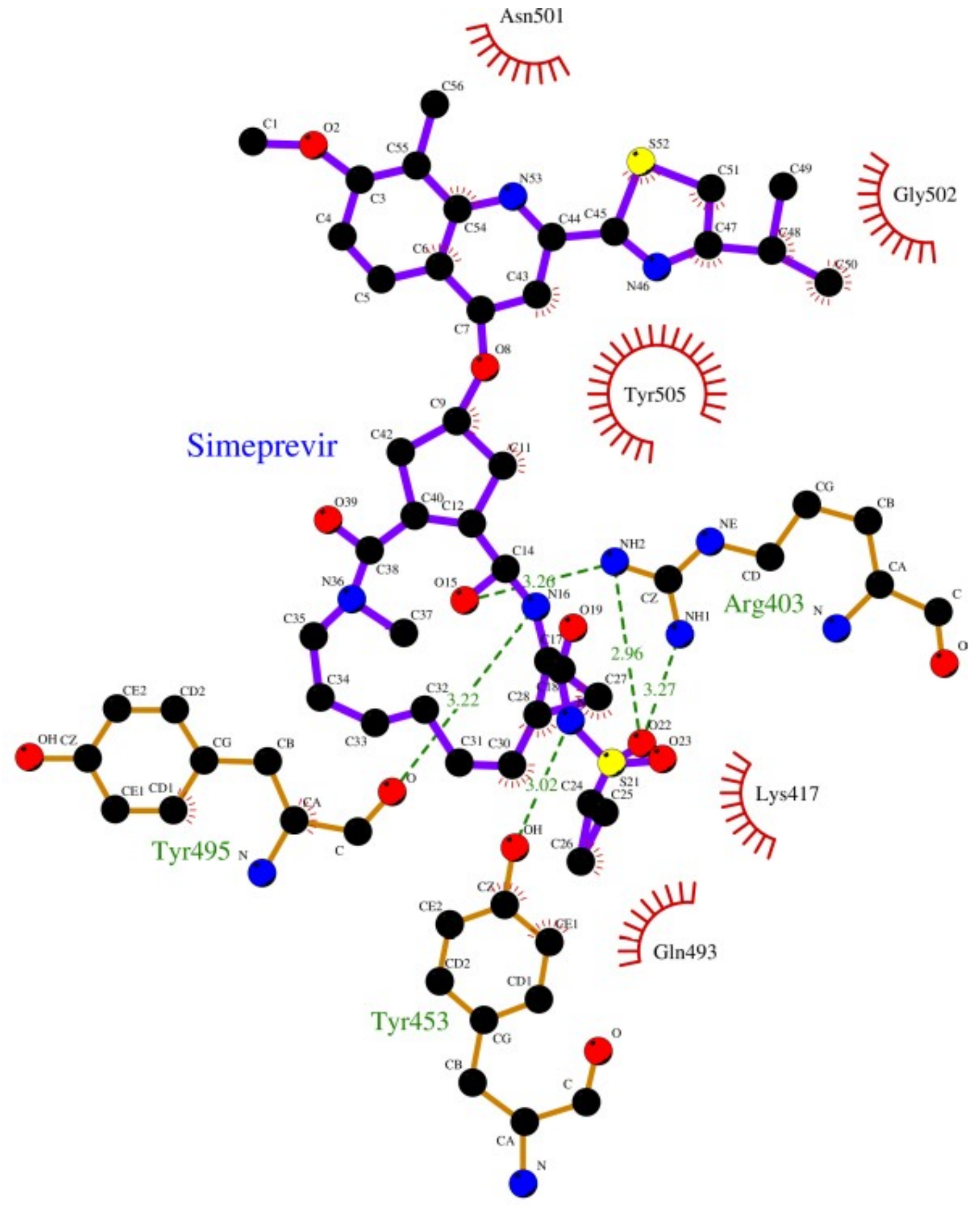

Fig. 16 The 2D contact map of simeprevir (ZINC000085540268) and RBD built in ligplot+. 


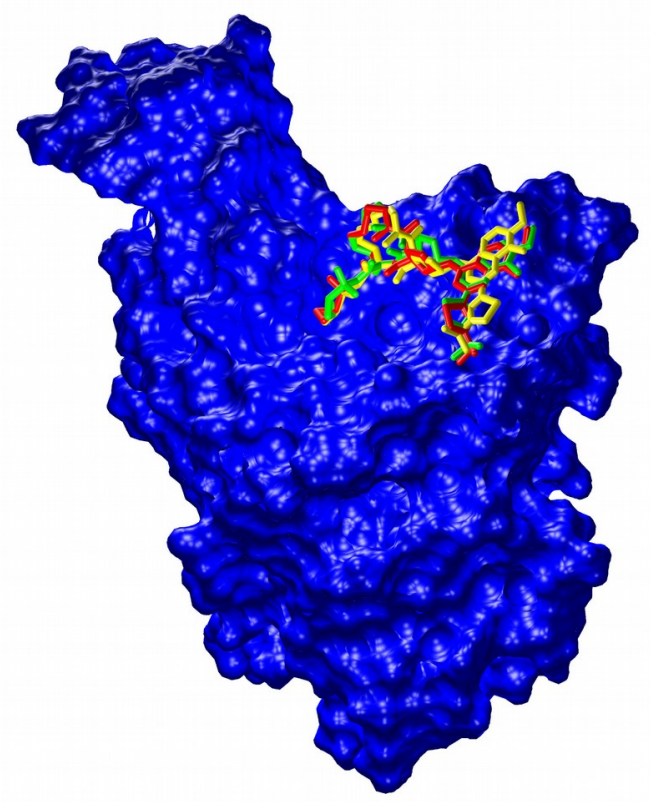

Fig. 17 The location of the different stereoisomers of simeprevir in the RBD binding site: red ZINC000085540268, yellow - ZINC000253632968, green - ZINC000150656835.

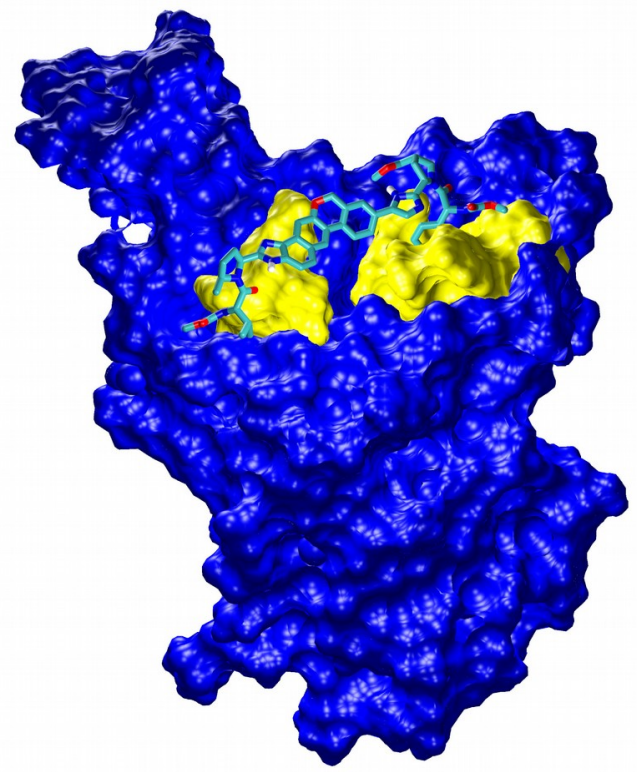

Fig. 18 The location of the velpatasvir binding site in RBD. 


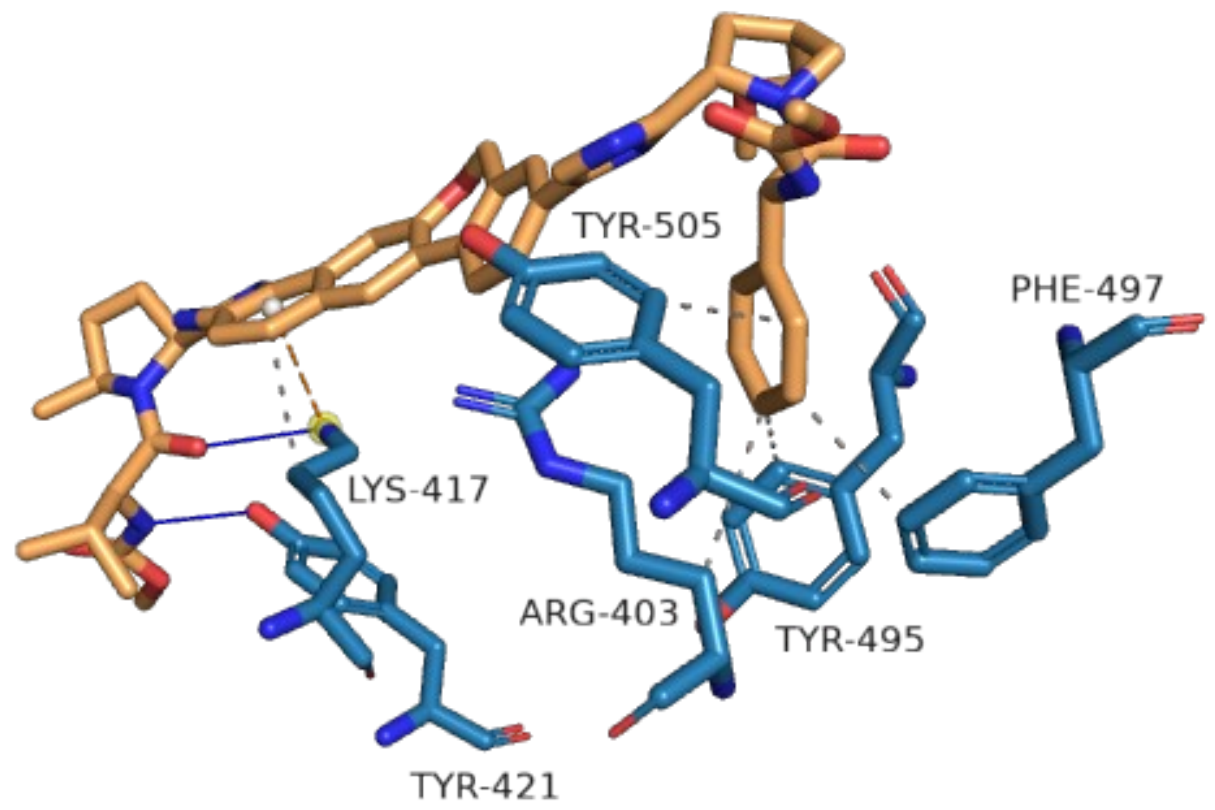

Fig. 19 The 3D contact map of velpatasvir and RBD built in PLIP tool. $\pi$-cation interaction is shown as red dotted line. 


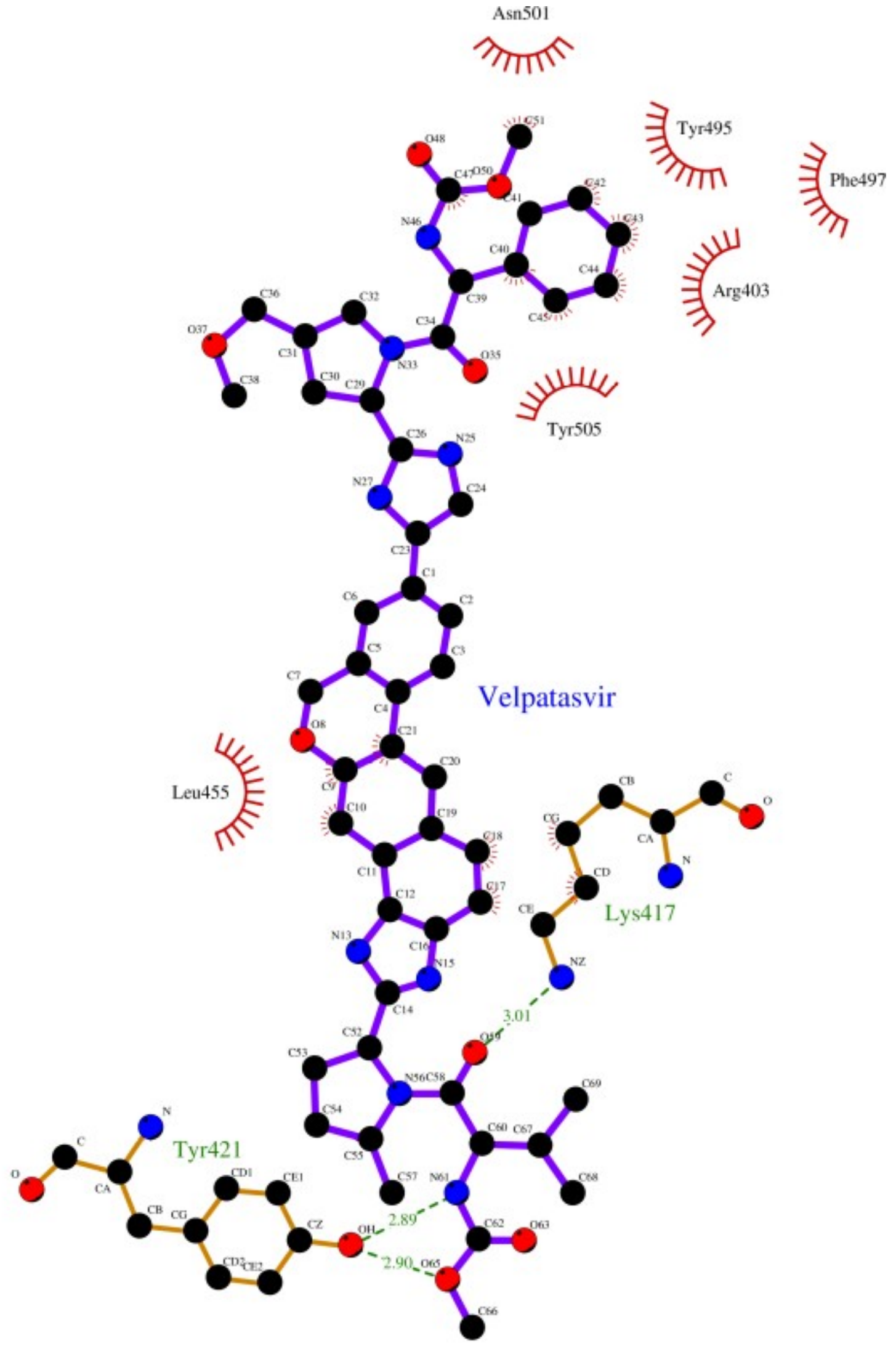

Fig. 20 The 2D contact map of velpatasvir and RBD built in ligplot+. 


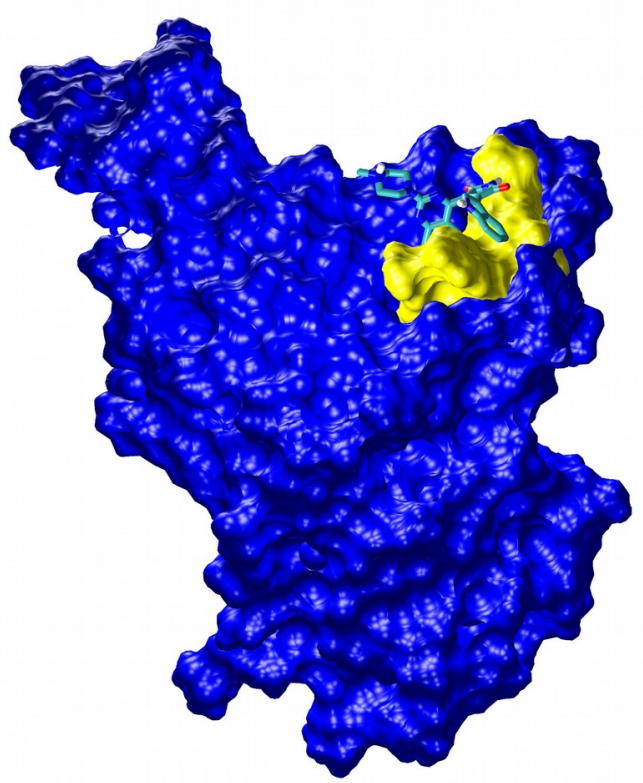

Fig. 21 The location of the sotrastaurin binding site in RBD.

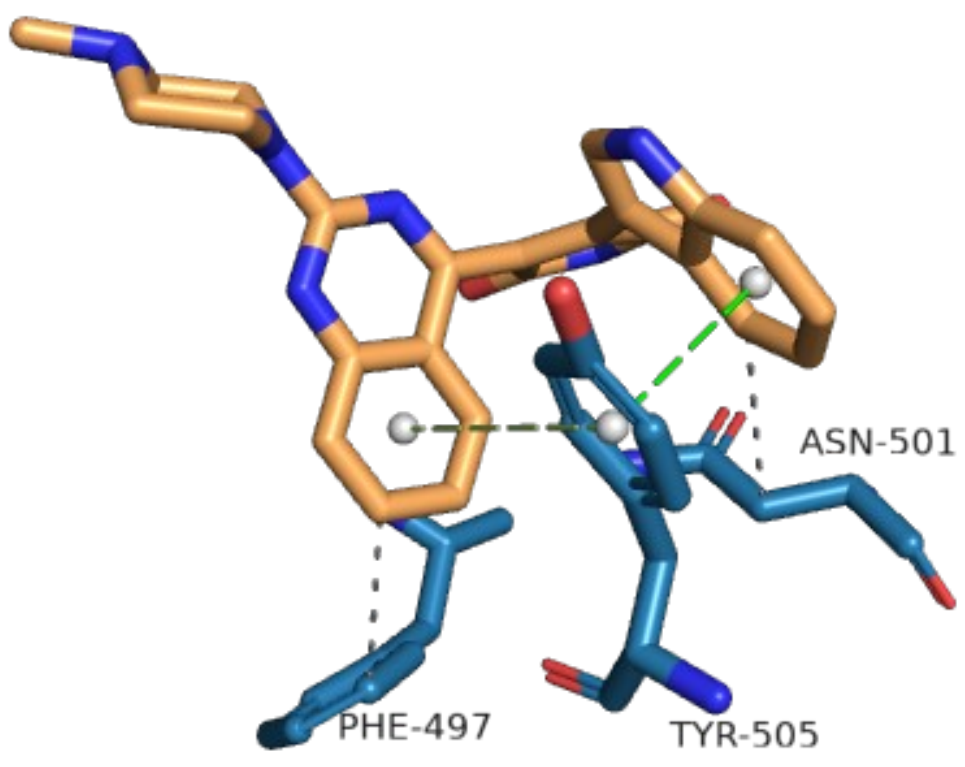

Fig. 22 The 3D contact map of sotrastaurin and RBD built in PLIP tool. 


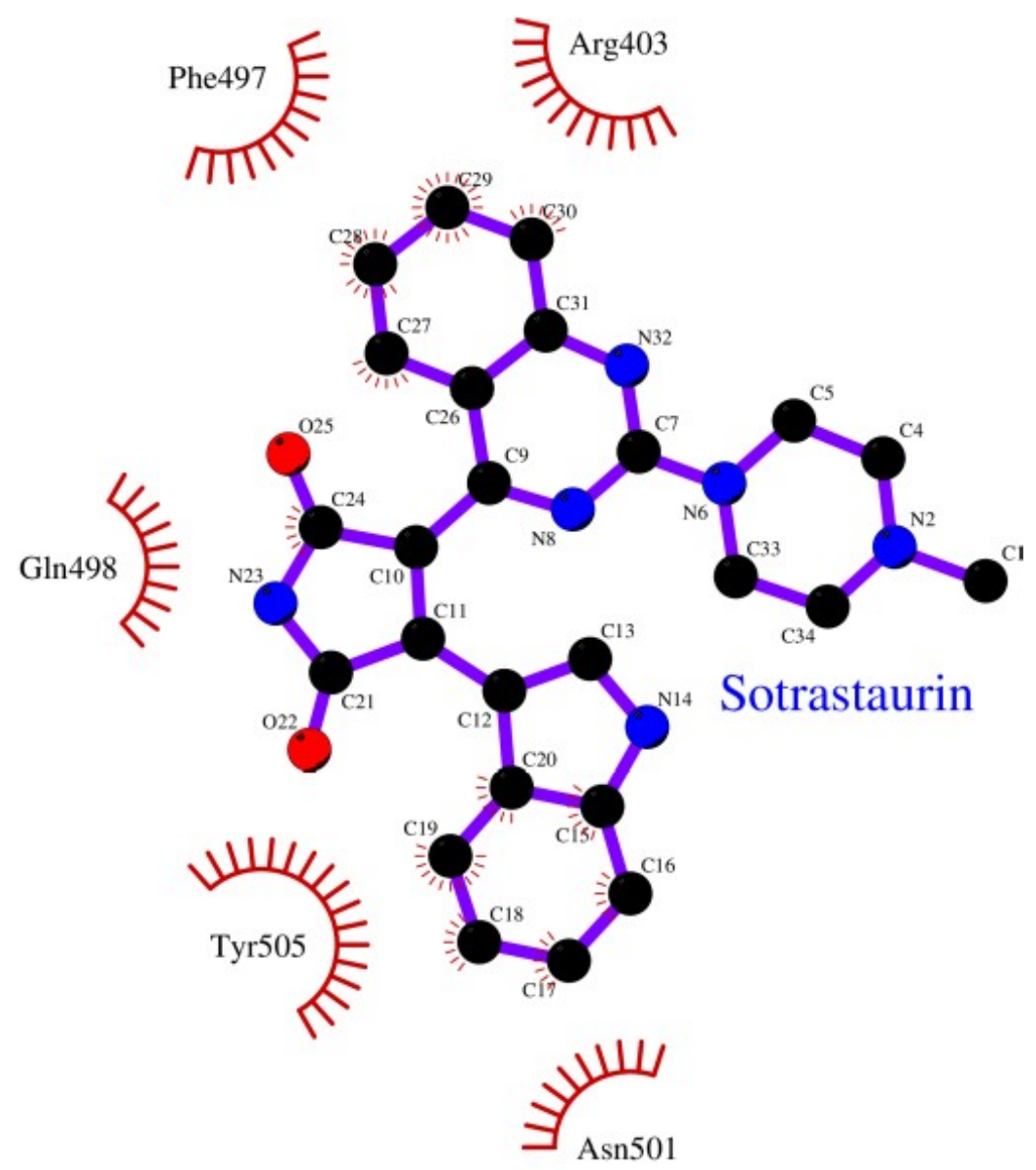

Fig. 23 The 2D contact map of sotrastaurin and RBD built in ligplot + .

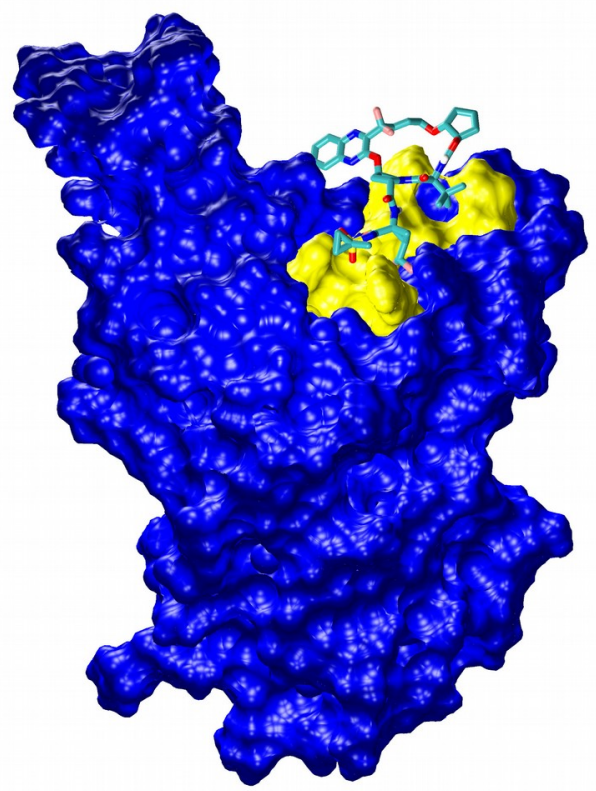

Fig. 24 The location of the glecaprevir binding site in RBD. 


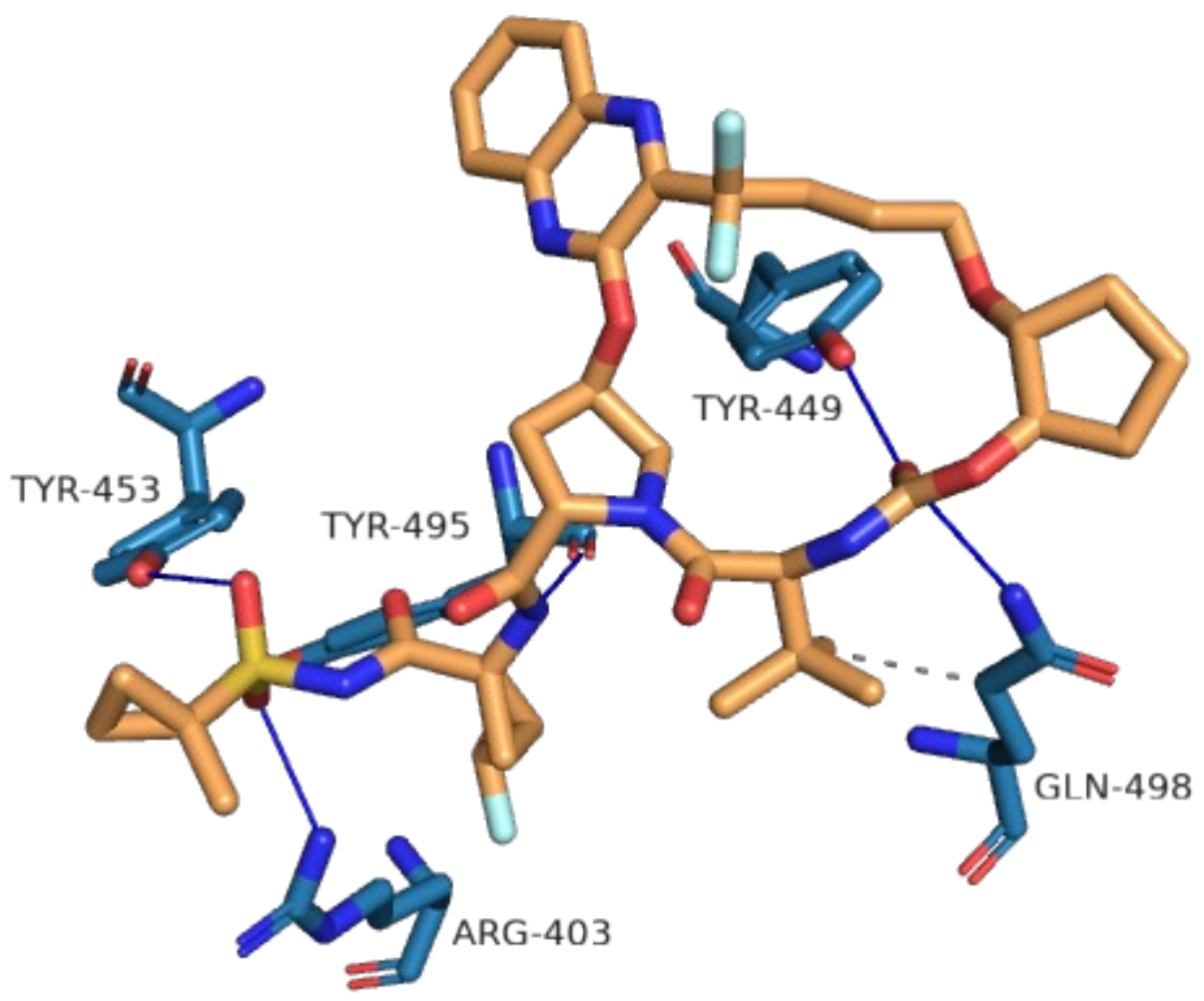

Fig. 25 The 3D contact map of glecaprevir and RBD built in PLIP tool. 


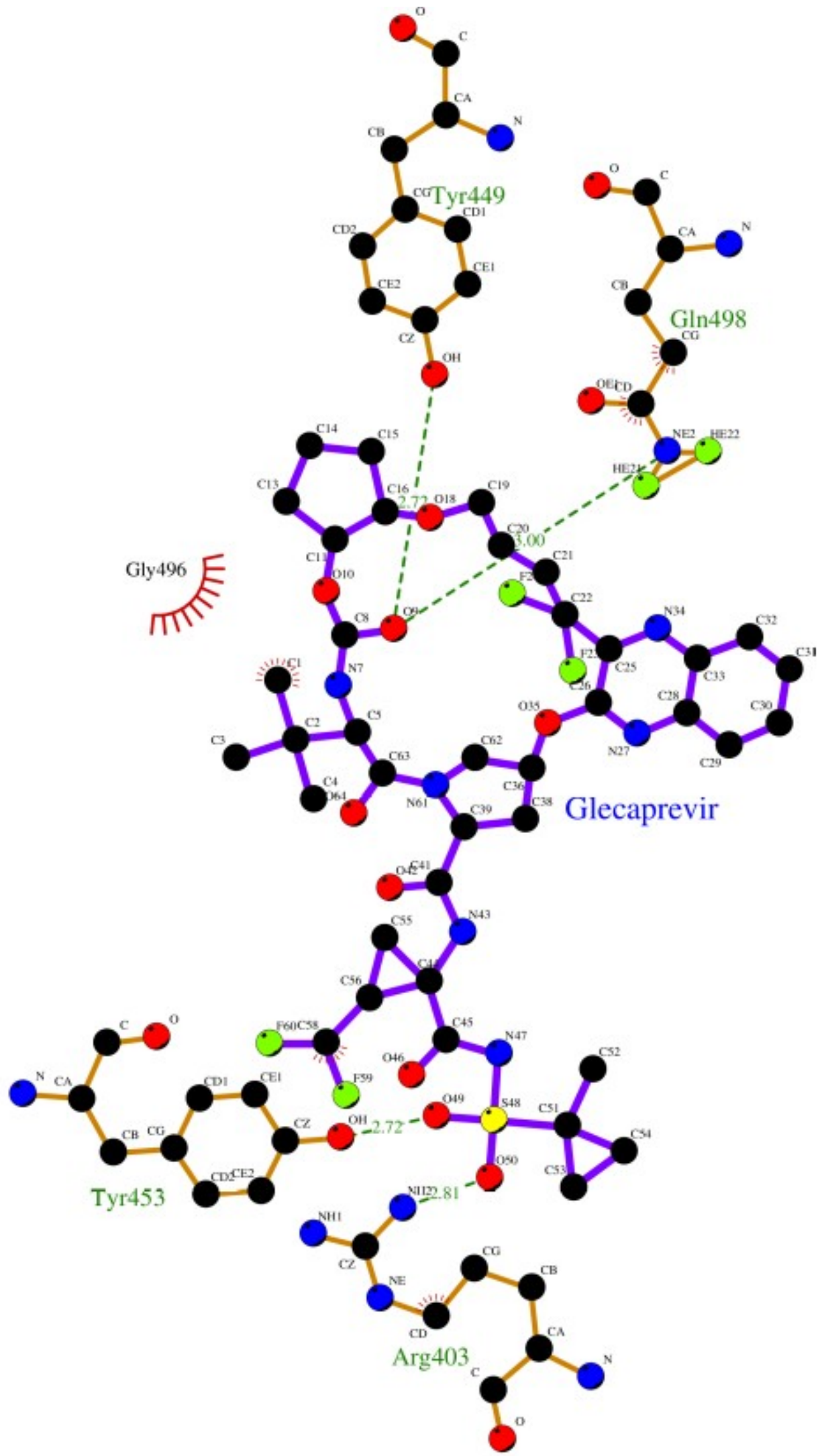

Fig. 26 The 2D contact map of glecaprevir and RBD built in ligplot+. 


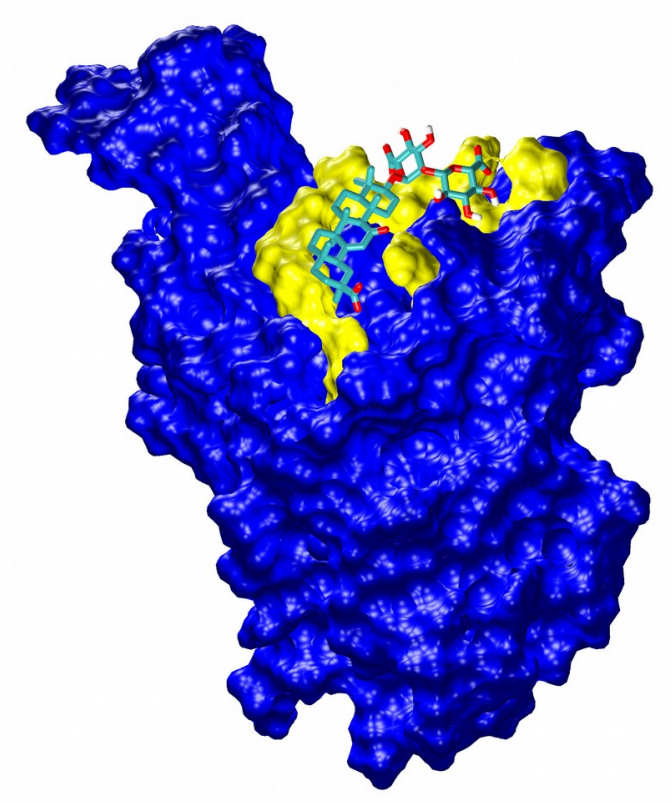

Fig. 27 The location of the alpha-glycyrrhizin binding site in RBD.

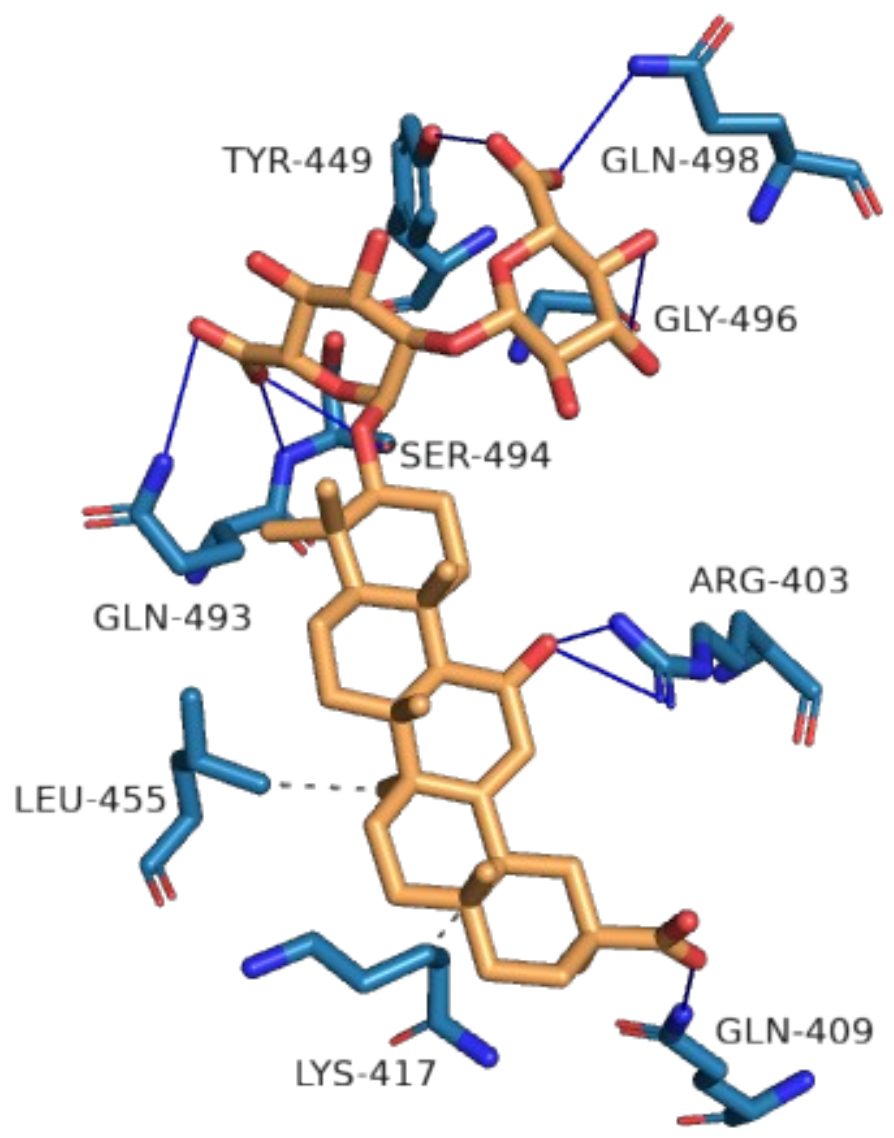

Fig. 28 The 3D contact map of alpha-glycyrrhizin and RBD built in PLIP tool. 


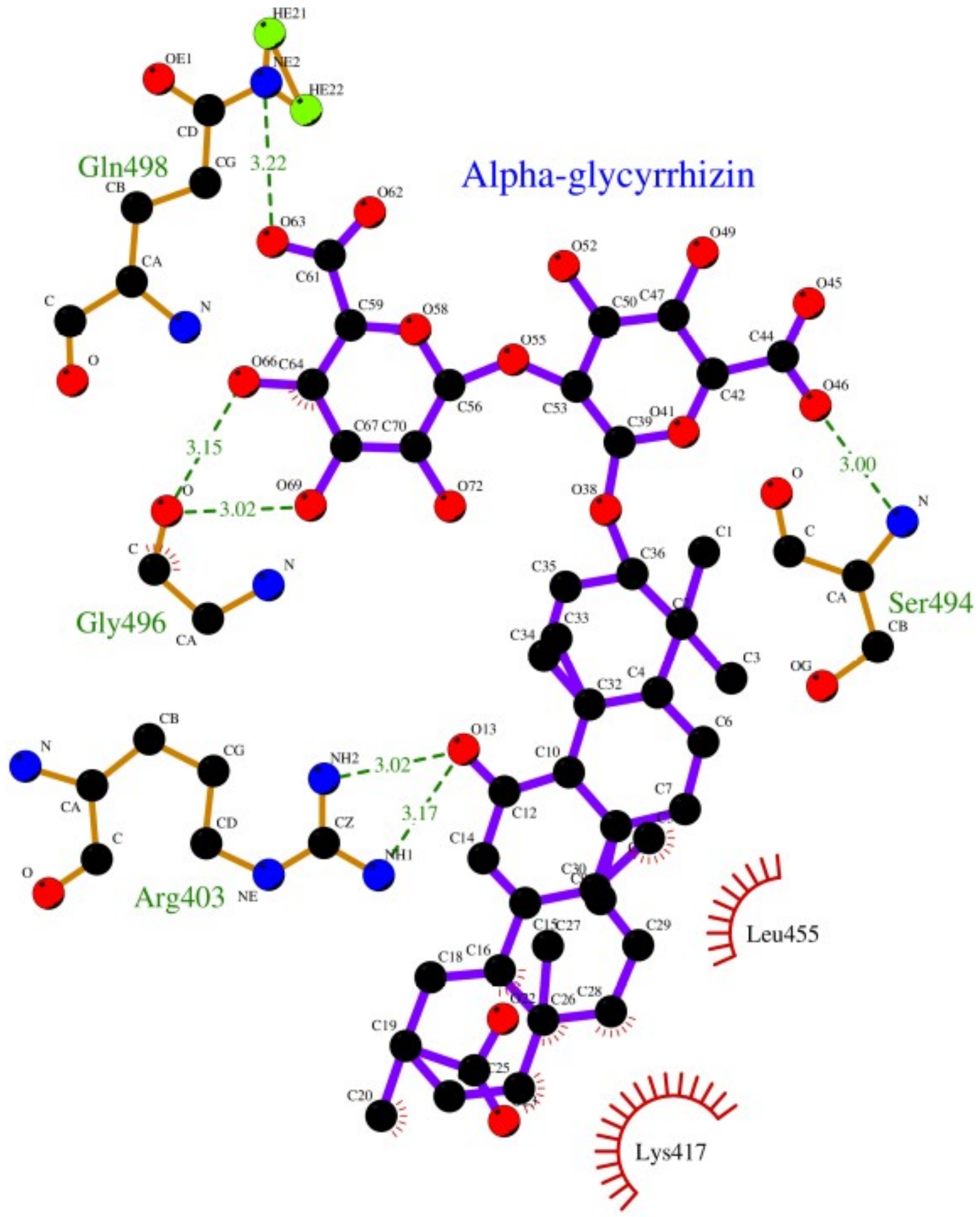

Fig. 29 The 2D contact map of alpha-glycyrrhizin and RBD built in ligplot+. 


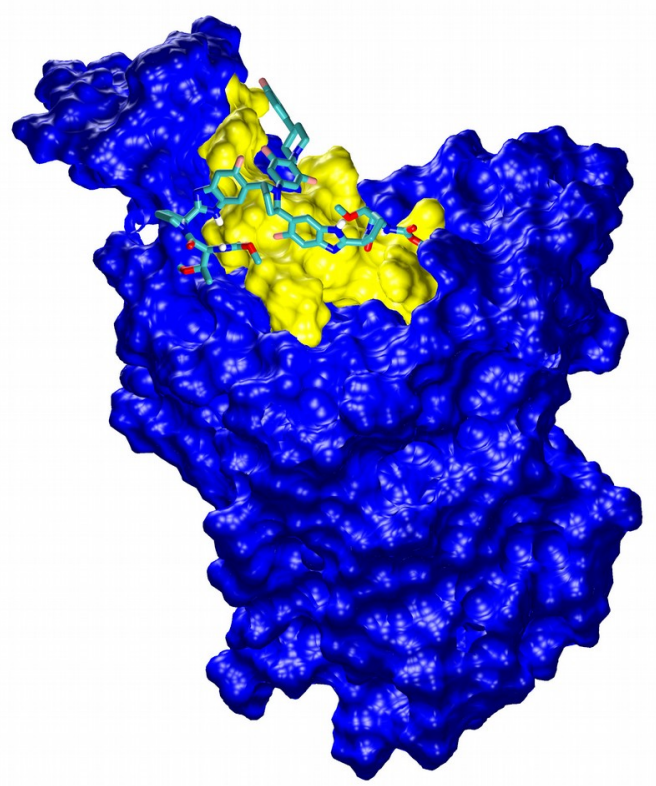

Fig. 30 The location of the pibrentasvir binding site in RBD.

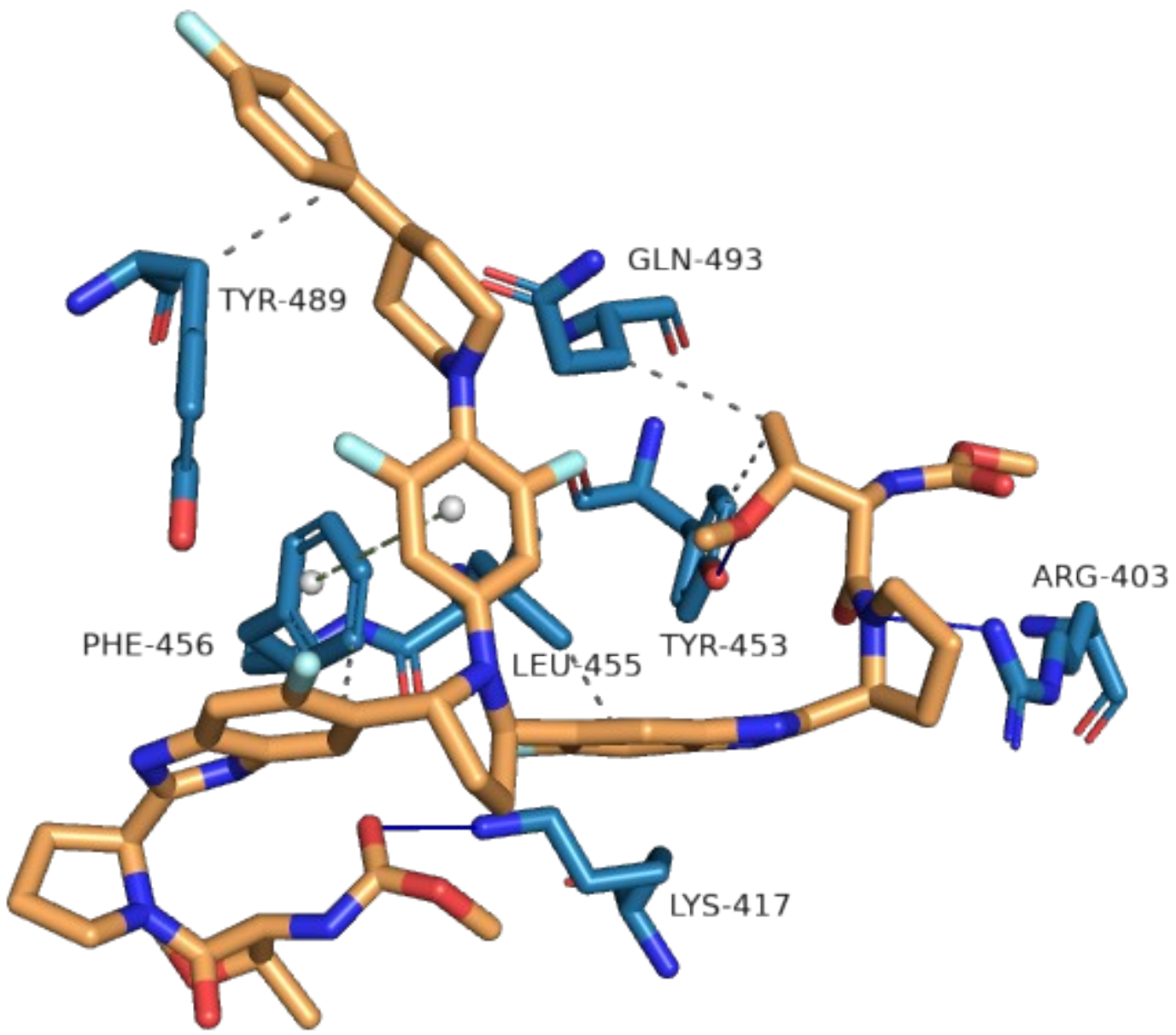

Fig. 31 The 3D contact map of pibrentasvir and RBD built in PLIP tool. 


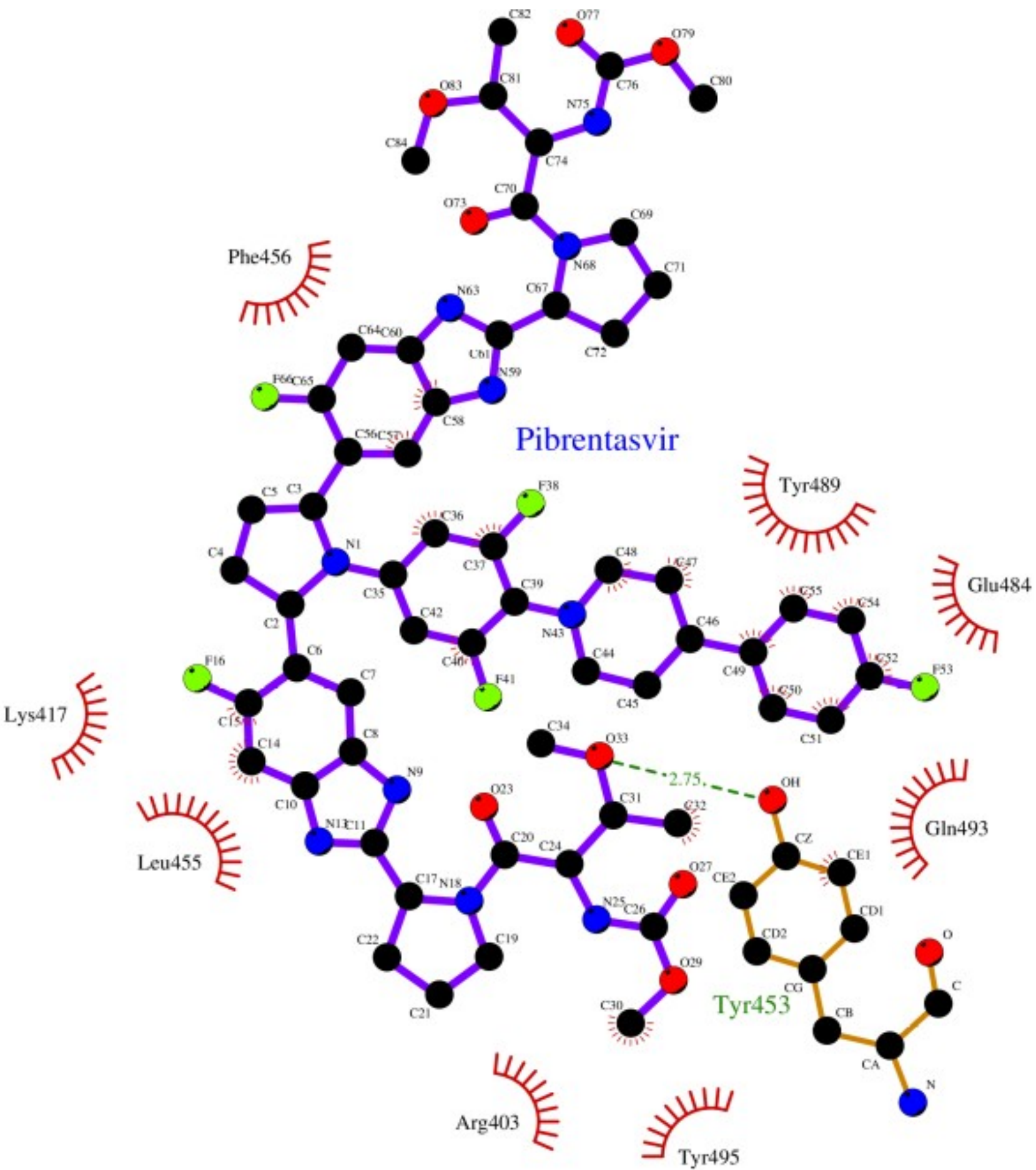

Fig. 32 The 2D contact map of pibrentasvir and RBD built in ligplot + . 


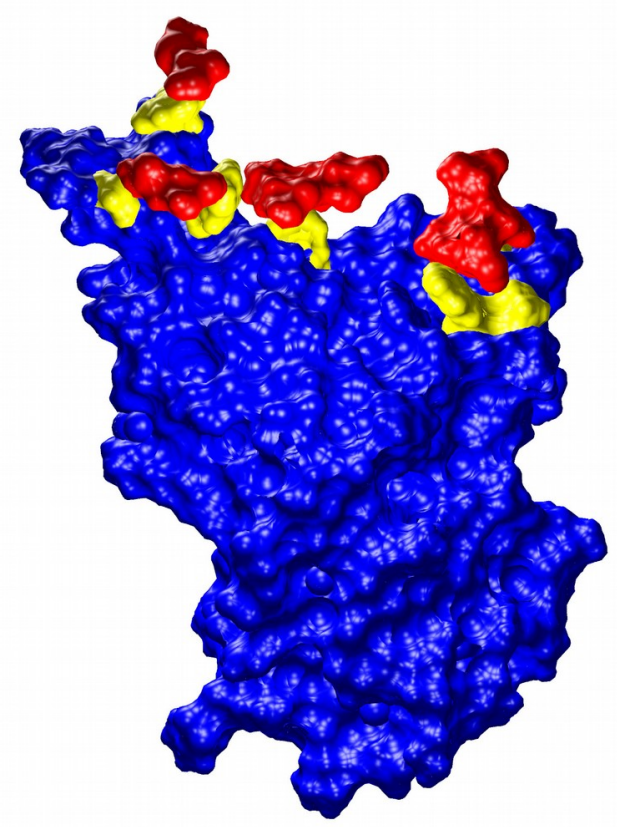

Fig. 33 The interaction of spike glycoprotein RBD (blue + yellow) with ACE2 (red). The residues of RBD that are in contact with ACE2 are shown in yellow. Coordinates were taken from the PDB ID $6 \mathrm{~m} 17$.

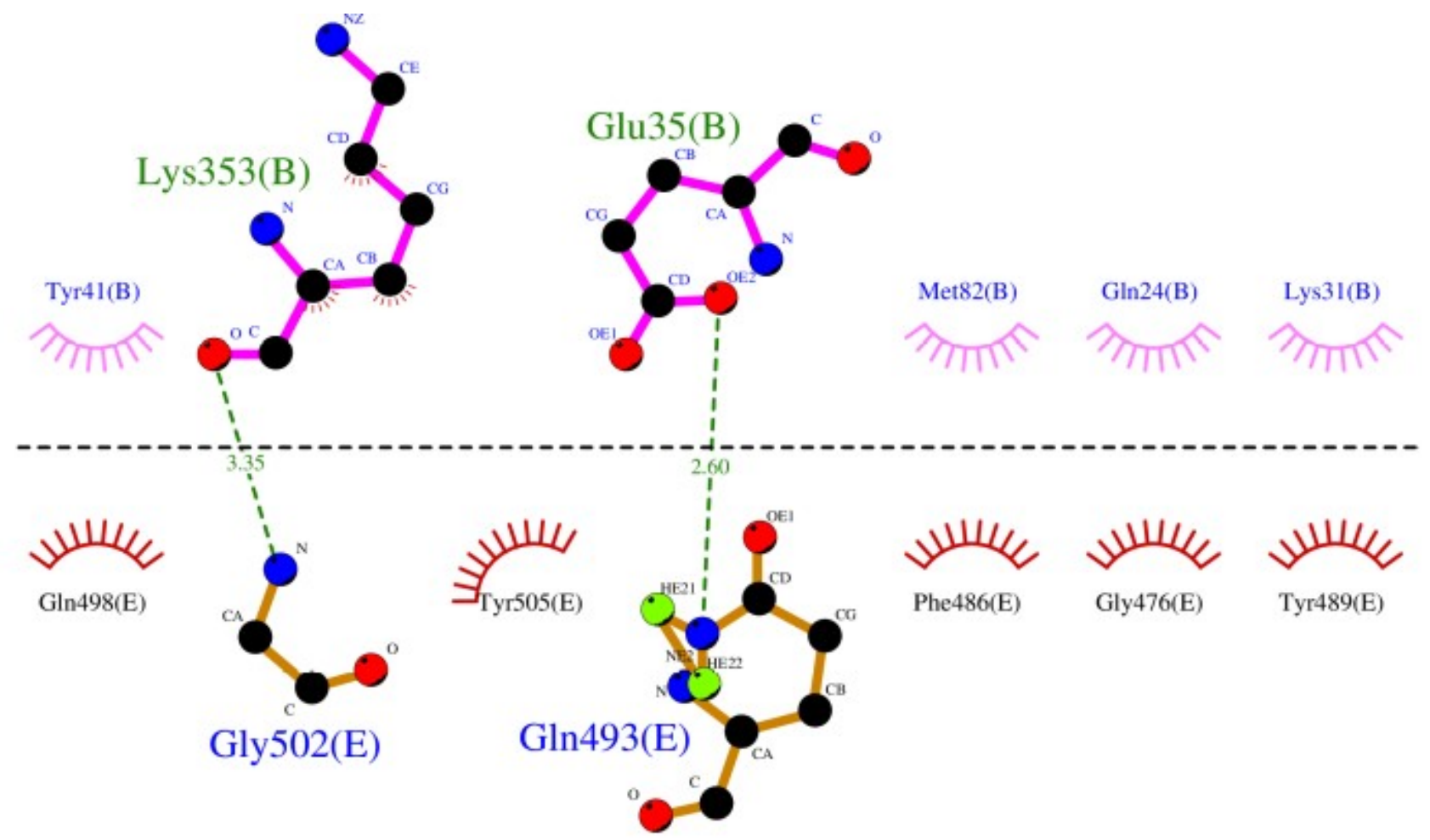

Fig. 34 The 2D contact map of spike glycoprotein RBD (chain E, bottom part of the figure) and ACE2 (chain B, upper part of the figure) built in ligplot + . Coordinates were taken from the PDB ID $6 \mathrm{~m} 17$. 


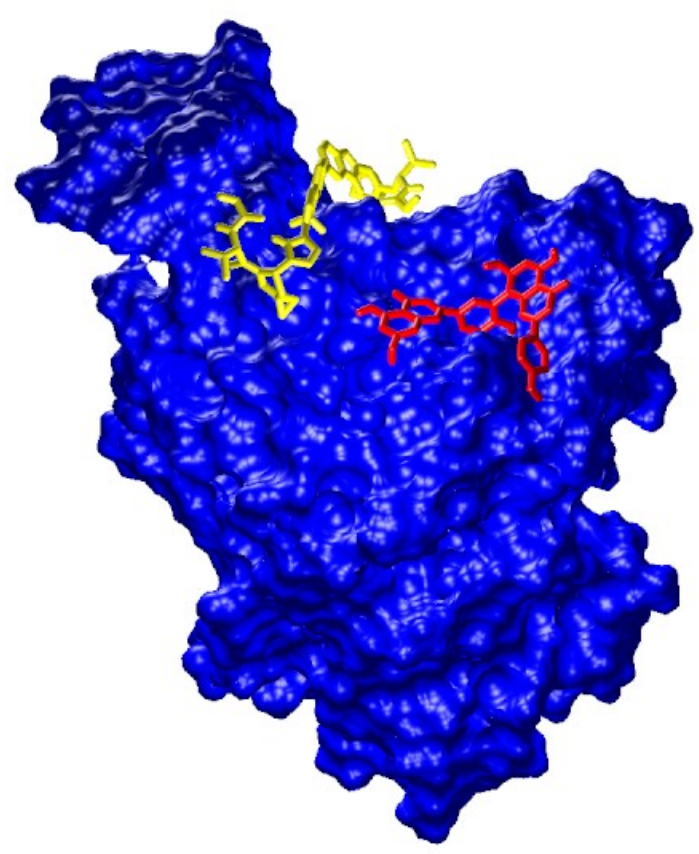

Fig. 35 The complex of spike glycoprotein RBD (blue) with amentoflavone (red) and ledipasvir (yellow).

\section{Conclusions}

We performed molecular docking of 248 drugs related to virus research against RBD of spike glycoprotein of SARS-CoV-2. The best-scored ligand is the biflavonoid amentoflavone. Most of the top-ranked ligands are drugs for HCV treatment. They are large hydrophobic molecules (M>700). The complexes of RBD and drugs are stabilized by hydrophobic interactions, hydrogen bonds and $\pi$ stacking. None of the drugs can overlap all the binding site residues of ACE2 on RBD. The two firstranked ligands (amentoflavone and ledipasvir) have different binding sites on RBD, so their combined use may be effective. Our results suggest that amentoflavone and ledipasvir are promising drug candidates for COVID-19 treatment. But before clinical trials, the careful testing is required. We encourage other researchers to explore the combination of amentoflavone and ledipasvir against SARS-CoV-2 in vitro and in vivo.

\section{Acknowledgements}

The molecular docking simulations were done using the facilities of GRID-cluster of O. Ya. Usikov Institute for Radiophysics and Electronics of NAS of Ukraine, Kharkiv, Ukraine.

\section{Conflict of Interest}

The authors declare that there is no conflict of interest.

\section{References}

1. https://www.arcgis.com/apps/opsdashboard/index.html\#/bda7594740fd40299423467b48e9ecf6

COVID-19 Dashbord by the Center for Systems Science and Engineering (CSSE) at Johns Hopkins University. Last updated at 5/27/2020, 1:32:40 PM. Accessed May 27, 2020.

2. Cascella, M., Rajnik, M., Cuomo, A., Dulebohn, S. C., \& Di Napoli, R. (2020). Features, Evaluation 
and Treatment Coronavirus (COVID-19) [Updated 2020 Apr 6]. In: StatPearls [Internet]. Treasure Island (FL): StatPearls Publishing; 2020 Jan-.Available from: https://www.ncbi.nlm.nih.gov/books/NBK554776/

3. Xu, X., Chen, P., Wang, J., Feng, J., Zhou, H., Li, X., ... Hao, P. (2020). Evolution of the novel coronavirus from the ongoing Wuhan outbreak and modeling of its spike protein for risk of human transmission. Science China. Life sciences, 63(3), 457-460. https://doi.org/10.1007/s11427-020-1637-5

4. Lu, R., Zhao, X., Li, J., Niu, P., Yang, B., Wu, H., ... Tan, W. (2020). Genomic characterisation and epidemiology of 2019 novel coronavirus: implications for virus origins and receptor binding. The Lancet 395(10224), 565-574. https://doi.org/10.1016/S0140-6736(20)30251-8

5. Zhang, H., Penninger, J. M., Li, Y., Zhong, N., \& Slutsky, A. S. (2020). Angiotensin-converting enzyme 2 (ACE2) as a SARS-CoV-2 receptor: molecular mechanisms and potential therapeutic target. Intensive care medicine, 46(4), 586-590. https://doi.org/10.1007/s00134-020-05985-9

6. Yan, R., Zhang, Y., Li, Y., Xia, L., Guo, Y., \& Zhou, Q. (2020). Structural basis for the recognition of SARS-CoV-2 by full-length human ACE2. Science (New York, N.Y.), 367(6485), 1444-1448. https://doi.org/10.1126/science.abb2762

7. Søndergaard, C. R., Olsson, M. H., Rostkowski, M., \& Jensen, J. H. (2011). Improved Treatment of Ligands and Coupling Effects in Empirical Calculation and Rationalization of pKa Values. Journal of chemical theory and computation, 7(7), 2284-2295. https://doi.org/10.1021/ct200133y

8. Olsson, M. H. M., Søndergaard, C. R., Rostkowski, M., \& Jensen, J. H. (2011). PROPKA3: consistent treatment of internal and surface residues in empirical pKa predictions. Journal of Chemical Theory and Computation 7(2), 525-537. https://doi.org/10.1021/ct100578z

9. Case, D. A., Ben-Shalom, I. Y., Brozell, S. R., Cerutti, D. S., Cheatham, T. E., III, Cruzeiro, V. W. D., ... Kollman, P. A. (2019). AMBER 2019, University of California, San Francisco.

10. Sanner M. F. (1999). Python: a programming language for software integration and development. Journal of molecular graphics and modelling, 17(1), 57-61.

11. Sterling, T., \& Irwin, J. J. (2015). ZINC 15 - Ligand Discovery for Everyone. Journal of chemical information and modeling, 55(11), 2324-2337. https://doi.org/10.1021/acs.jcim.5b00559

12. Kim, S., Chen, J., Cheng, T., Gindulyte, A., He, J., He, S., ... Bolton, E. E. (2019). PubChem 2019 update: improved access to chemical data. Nucleic acids research, 47(D1), D1102-D1109. https://doi.org/10.1093/nar/gky1033

13. O'Boyle, N. M., Banck, M., James, C. A., Morley, C., Vandermeersch, T., \& Hutchison, G. R. (2011). Open Babel: An open chemical toolbox. Journal of cheminformatics, 3, 33. https://doi.org/10.1186/1758-2946-3-33

14. Trott, O., \& Olson, A. J. (2010). AutoDock Vina: improving the speed and accuracy of docking with a new scoring function, efficient optimization, and multithreading. Journal of computational chemistry, 31(2), 455-461. https://doi.org/10.1002/jcc.21334

15. Humphrey, W., Dalke, A., \& Schulten, K. (1996). VMD: visual molecular dynamics. Journal of molecular graphics, 14(1), 33-28. https://doi.org/10.1016/0263-7855(96)00018-5

16. Salentin, S., Schreiber, S., Haupt, V. J., Adasme, M. F., \& Schroeder, M. (2015). PLIP: fully automated protein-ligand interaction profiler. Nucleic acids research, 43(W1), W443-W447. https://doi.org/10.1093/nar/gkv315

17. Laskowski, R. A., \& Swindells, M. B. (2011). LigPlot+: multiple ligand-protein interaction diagrams for drug discovery. Journal of chemical information and modeling, 51(10), 2778-2786. https://doi.org/10.1021/ci200227u

18. Yu, S., Yan, H., Zhang, L., Shan, M., Chen, P., Ding, A., \& Li, S. F. (2017). A Review on the 
Phytochemistry, Pharmacology, and Pharmacokinetics of Amentoflavone, a Naturally-Occurring

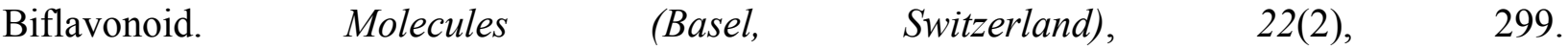
https://doi.org/10.3390/molecules22020299

19. Kimura, Y., Ito, H., Ohnishi, R., \& Hatano, T. (2010). Inhibitory effects of polyphenols on human cytochrome P450 3A4 and 2C9 activity. Food and chemical toxicology : an international journal published for the British Industrial Biological Research Association, 48(1), 429-435. https://doi.org/10.1016/j.fct.2009.10.041

20. Pan, X., Tan, N., Zeng, G., Zhang, Y., \& Jia, R. (2005). Amentoflavone and its derivatives as novel natural inhibitors of human Cathepsin B. Bioorganic \& medicinal chemistry, 13(20), 5819-5825. https://doi.org/10.1016/j.bmc.2005.05.071

21. Gottwein, J. M., Pham, L. V., Mikkelsen, L. S., Ghanem, L., Ramirez, S., Scheel, T., Carlsen, T., \& Bukh, J. (2018). Efficacy of NS5A Inhibitors Against Hepatitis C Virus Genotypes 1-7 and Escape Variants. Gastroenterology, 144(5), 1435-1448. https://doi.org/10.1053/j.gastro.2017.12.015

22. McCauley, J. A., \& Rudd, M. T. (2016). Hepatitis C virus NS3/4a protease inhibitors. Current opinion in pharmacology, 30, 84-92. https://doi.org/10.1016/j.coph.2016.07.015

23. Lamb Y. N. (2017). Glecaprevir/Pibrentasvir: First Global Approval. Drugs, 77(16), 1797-1804. https://doi.org/10.1007/s40265-017-0817-y

24. Mevada, V., Dudhagara, P., Gandhi, H., Vaghamshi, N., Beladiya, U., \& Patel, R. (2020). Drug Repurposing of Approved Drugs Elbasvir, Ledipasvir, Paritaprevir, Velpatasvir, Antrafenine and Ergotamine for Combating COVID19 (Version 2). ChemRxiv. https://doi.org/10.26434/chemrxiv.12115251.v2

25. Hosseini, F. S., \& Amanlou, M. (2020). Simeprevir, Potential Candidate to Repurpose for Coronavirus Infection: Virtual Screening and Molecular Docking Study. Preprints, 2020, 2020020438. https://doi.org/10.20944/preprints202002.0438.v1

26. Kouznetsova, V., Huang, D., \& Tsigelny, I. F. (2020, April 9). Potential COVID-19 Protease Inhibitors: Repurposing FDAapproved Drugs (Version 1). ChemRxiv. https://doi.org/10.26434/chemrxiv.12093900.v1

27. Balasubramaniam, M., \& Shmookler Reis, R. (2020, April 7). Computational Target-Based Drug Repurposing of Elbasvir, an Antiviral Drug Predicted to Bind Multiple SARS-CoV-2 Proteins (Version 2). ChemRxiv. https://doi.org/10.26434/chemrxiv.12084822.v2

28. Chen, Y. W., Yiu, C. B., \& Wong, K. Y. (2020). Prediction of the SARS-CoV-2 (2019-nCoV) 3Clike protease (3CL pro) structure: virtual screening reveals velpatasvir, ledipasvir, and other drug repurposing candidates. F1000Research, 9, 129. https://doi.org/10.12688/f1000research.22457.2

29. Anwar, M. U., Adnan, F., Abro, A., Khan, M. R., Rehman, A. U., Osama, M., ... Assir, M. Z. (2020, May 4). Combined Deep Learning and Molecular Docking Simulations Approach Identifies Potentially Effective FDA Approved Drugs for Repurposing Against SARS-CoV-2 (Version 1). ChemRxiv. https://doi.org/10.26434/chemrxiv.12227363.v1

30. Chen, H., Zhang, Z., Wang, L., Huang, Z., Gong, F., Li, X., Chen, Y., \& WU, J. J. (2020). First clinical study using HCV protease inhibitor danoprevir to treat naive and experienced COVID-19 patients. MedRxiv 2020.03.22.20034041. https://doi.org/10.1101/2020.03.22.20034041

31. Evenou, J. P., Wagner, J., Zenke, G., Brinkmann, V., Wagner, K., Kovarik, J., ... Baier, G. (2009). The potent protein kinase C-selective inhibitor AEB071 (sotrastaurin) represents a new class of immunosuppressive agents affecting early T-cell activation. The Journal of pharmacology and experimental therapeutics, 330(3), 792-801. https://doi.org/10.1124/jpet.109.153205

32. Chang, G., Zheng, J., Xiao, W., Chang, S., Wei, Q., Wu, H., ... Shi, J. (2018). PKC inhibition of sotrastaurin has antitumor activity in diffuse large B-cell lymphoma via regulating the expression of MCT-1. Acta biochimica et biophysica Sinica, 50(4), 399-407. https://doi.org/10.1093/abbs/gmy021 
33. Matz, M., Naik, M., Mashreghi, M. F., Glander, P., Neumayer, H. H., \& Budde, K. (2011). Evaluation of the novel protein kinase $\mathrm{C}$ inhibitor sotrastaurin as immunosuppressive therapy after renal transplantation. Expert opinion on drug metabolism \& toxicology, 7(1), 103-113. https://doi.org/10.1517/17425255.2011.540238

34. Ming, L. J., \& Yin, A. C. Y. (2013). Therapeutic Effects of Glycyrrhizic Acid. Natural Product Communications, 8(3), 415-418.

35. Li, J. Y., Cao, H. Y., Liu, P., Cheng, G. H., \& Sun, M. Y. (2014). Glycyrrhizic acid in the treatment of liver diseases: literature review. BioMed research international, 2014, 872139. https://doi.org/ $\underline{10.1155 / 2014 / 872139}$

36. https://www.cebm.net/covid-19/registered-trials-and-analysis/ Accessed May 27, 2020. 